TRANSACTIONS OF THE

AMERICAN MATHEMATICAL SOCIETY

Volume 312, Number 1, March 1989

\title{
DETERMINACY OF SUFFICIENTLY DIFFERENTIABLE MAPS
}

\author{
ALAN M. SELBY
}

\begin{abstract}
Variants of the algebraic conditions of Mather are shown to be sufficient for the $k$-determinacy of $C^{u}$ maps with respect to $j$-flat, contact (or right) $C^{r}$ equivalence relations where $u-k \leq r \leq u-k+j+1$ and $0 \leq j<k \leq u$. The required changes of coordinates and matrix-valued functions are constructed from the variation of coefficients in polynomials. The main result follows from a finite-dimensional, polynomial pertubation argument which employs a parameter-dependent polynomial representation of functions based on Taylor's formula. For $r>k$, the algebraic conditions are seen to be necessary.
\end{abstract}

\section{INTRODUCTION}

Algebraic conditions for the $k$-determinacy of $C^{\infty}$ mappings, or rather the germs thereof, with respect to $C^{\infty}$ contact and right equivalence relations are given in Mather [1969a]. They have since been reproduced in several introductory texts. In Deakin [1977], a $C^{\infty}$ real-valued map-germ $f: R^{n} \rightarrow R$ which satisfies Mather's algebraic condition for right $k$-determinacy is said to be $k$-complete. Deakin [1977] shows by a tangent space (or directional derivative) argument that right $k$-determinacy with differentiable dependence on a small parameter implies $(k+1)$-completeness. This gives a partial converse to Mather's result. The 1974 lecture notes in Martinet [1982] in an exercise observe that the algebraic condition of Mather for right $k$-determinacy is sufficient for a local form of $(k-1)$-determinacy. In the exercise, the distance between two function germs is given by the distance between their respective $k$-jets. A tangent space argument clearly implies the converse. Hence for germs of $C^{\infty}$ maps $f: R^{n} \rightarrow R, k$-completeness is necessary and sufficient for the local form of $(k-1)$-determinacy described in Martinet [1982].

Elliptic-like coercive conditions for a $C^{0}$, alias topological, right $k$-determinacy of $C^{\infty}$ mappings are given in Kuo [1969a]. In Takens [1971] these ellipticlike coercive conditions of Kuo [1969a] are generalized to obtain $k$-determinacy results for a $C^{r}$ right equivalence relation between sufficiently differentiable

Received by the editors January 1, 1985 and, in revised form, January 1, 1988.

1980 Mathematics Subject Classification (1985 Revision). Primary 58C27.

Key words and phrases. Algebraic conditions for $C^{r}$ contact and right determinacy, Taylor's formula, polynomial representation of maps, ordinary differential equations. 
maps. The best results with coercive conditions appear to be in Lefebvre and Pourprix [1984]. The latter paper presents coercive conditions sufficient for the $k$-determinacy of $C^{u}$ maps with respect to two $j$-flat, $C^{r}$ contact equivalence relations.

The $k$-jet of a map of a $C^{k}$ or smoother map $f: R^{n} \rightarrow R^{m}$ is here identified with the sum of terms of order $k$ or less in the Taylor series expansion of $f$ about the origin $x=0$ of its domain. We say the map $f$ satisfies the algebraic conditions of Mather for right and contact $k$-determinacy of $C^{\infty}$ maps iff its $k$-jet satisfies the conditions.

In the present article, variants $A R_{k, j}(f)$ and $A C_{k, j}(f)$ of the algebraic conditions of Mather for right and contact $k$-determinacy are shown to be sufficient for local and nonlocal forms of $(k-1)$-determinacy with respect to $C^{r}, j$-flat, equivalence relations between $C^{u}$ maps where $u-k \leq r \leq u-$ $k+j+1$. For $r>k$, converse results hold by tangent space arguments. The algebraic conditions are seen to be necessary.

This article is composed as follows. $\S 2$ gives a few definitions. $\S 3$ states the results and indicates the proofs of all assertions but for the main determinacy result Theorem 3.1. Preparations for its proof are given in $\S \S 4$ and 5. The proof is presented in $\S 6$.

$\S 4$ in particular presents in two lemmas, Lemmas 4.1 and 4.2 , the $k$ th-order polynomial approximations that are consequences of the algebraic conditions. These polynomial approximations establish the contact and right determinacy results in this article, modulo terms of order $k$. The demonstrations of the lemmas might be skipped on first-reading. $\S 5$ represents each $C^{k}$ or smoother map by a parameter-dependent family of polynomials of degree $k$. The representation is based on Taylor's formula. The representation reproduces the original map when the parameter coincides with the argument of the polynomial. This map reproducing property and a further diagonal-value reproducing property are also described in $\S 5$.

The proof of Theorem 3.1 given in $\S 6$ combines the $k$ th-order polynomial approximations in $\S 4$ and the polynomial representation in $\S 5$ to obtain a $k$ determinacy theorem via a finite-dimensional polynomial perturbation argument. The proof is a consequence of the ordinary, that is not generalized, implicit function theorem. The initial model for this proof was the generalized Morse lemma of Hormander [1971] as reproduced in Nirenberg [1973-1974] and the two-timing or two-variable method in perturbation theory as described in Nayfeh [1973]. Further remarks on the origin of the present article are given in $\S 7$ and at various other points below.

\section{DEFINITIONS}

Two $R^{m}$-valued functions $f(x)$ and $g(x)$ of $x$ in $R^{n}$ are said to be $j$-flat, $C^{r}$ contact equivalent at the origin iff the following hold. 
(1) there is a $C^{r}$ change of variables on the domain space $R^{n}, y(x)=$ $x+O\left(|x|^{j+1}\right) ;$ and

(2) there is a $m \times m$ matrix-valued $C^{r}$ function $A(x)$ with $A(x)=I_{m}+$ $O\left(|x|^{j+1}\right)$ and $A(0)$ nonsingular

such that $A(x) \cdot f(y(x))=g(x)$ on some neighbourhood of the origin.

The change of variables $y(x)$ required above differs from the identity map $I(x)=x$ by a $j$-flat perturbation. That is, a function of the form $O\left(|x|^{j+1}\right)$ as $x \rightarrow 0$. The required matrix $A(x)$ also differs from the $m \times m$ identity matrix $I_{m}$ by a $j$-flat perturbation. Note $y(x)$ fixes the origin (that is, $y(0)=0$ ). Also if $j>0$ then $A(0)$ coincides with the identity matrix $I_{m}$.

Two $R^{m}$-valued functions $f(x)$ and $g(x)$ of $x$ in $R^{n}$ are said to be $j$-flat, $C^{r}$ right equivalent iff there is a $C^{r}$ change of variables $y(x)=x+O\left(|x|^{j+1}\right)$ on the domain space $R^{n}$ such that $f(y(x))=g(x)$ on some neighbourhood of the origin. This is the special case given by $A(x)=I_{m}$ identically, of the $C^{r}$, $j$-flat contact equivalence relation.

A $C^{u}$ map $f$ is said to be $j$-flat, contact (or right) $C^{r} k$-determined at $x=0$ in $R^{n}$ iff each of its $C^{u}$ perturbations $g(x)=f(x)+h(x)$ are $j$-flat, $C^{r}$ contact (respectively right) equivalent to the given map $f$ on a neighbourhood of the origin when the disturbance $h(x)=O\left(|x|^{k+1}\right)$ is $C^{k}$ and of order $k+1$.

A $C^{u}$ map is finitely determined with respect to the $j$-flat, $C^{r}$ contact (or right) equivalence relation iff it is $C^{r} j$-flat, contact (resp., right) $k$-determined whenever $k \leq u$ is sufficiently large.

\section{RESUltS}

Notation. Let $j$ and $k$ be integers with $0 \leq j<k$. Let $u \geq k$ denote a positive integer, $+\infty$ or $\omega$. Let $m$ and $n$ denote positive integers. Let $R$ denotes the field of real (or complex) numbers. For each multi-index $\alpha=\left(\alpha_{1}, \ldots, \alpha_{n}\right)$ with nonnegative integer components, let

$$
\partial^{\alpha}=\left[\partial / \partial x_{1}\right]^{\alpha_{1}} \cdots\left[\partial^{\alpha_{n}} / \partial x_{n}\right]^{\alpha_{n}}
$$

be a mixed, partial differential operator of order $q=|\alpha|=\alpha_{1}+\cdots+\alpha_{n}$. The factorial of the multi-index $\alpha$ is $(\alpha) !=\left(\alpha_{1}\right) ! \cdot\left(\alpha_{2}\right) ! \cdots\left(\alpha_{n}\right) !$. The corresponding multinomial coefficient is

$$
\left(\begin{array}{l}
q \\
\alpha
\end{array}\right)=\frac{q !}{(\alpha) !}=\frac{q !}{\left(\alpha_{1}\right) ! \cdot\left(\alpha_{2}\right) ! \cdots\left(\alpha_{n}\right) !}
$$

The corresponding monomial of order $|\alpha|$ is $x^{\alpha}=x_{1}^{\alpha_{1}} x_{2}^{\alpha_{2}} \cdots x_{n}^{\alpha_{n}}$.

Chain-rule, $k$-jet operators and Jacobians. Let $f$ be a $C^{u}$ map of an open neighbourhood of the origin $x=0$ in the space $R^{n}$ with values in the space $R^{m}$. The polynomial $k$-jet of $f(x)$ is denoted by

$$
p(x)=J_{k} f(x) \stackrel{\text { def }}{=} \sum_{|\alpha| \leq k} \frac{1}{(\alpha) !} \partial^{\alpha} f(0) \cdot x^{\alpha}
$$


below. The sum involves all terms in the Taylor series of $f$ at the origin up to and including the terms of order $k$. The Jacobian matrix of the $k$-jet $p$ is denoted by $D_{x} p(x)$. Its components are polynomials.

The iterated chain-rule says $J_{k}\{u \circ v\}=J_{k}\left\{\left(J_{k} u\right) \circ\left(J_{k} v\right)\right\}$ whenever $u: R^{m} \rightarrow$ $R^{n}$ and $v: R^{p} \rightarrow R^{n}$ are polynomials or $C^{k}$ maps with $v(0)=0$. Further, the product rule implies $J_{k}\{B(u, v)\}=J_{k}\left\{B\left(J_{k} u, J_{k} v\right)\right\}$ if $B(u, v)$ is a bilinear form and both $u$ and $v$ are polynomials or $C^{k}$ maps. These observations are useful in many calculations. In particular, they allow the insertion or omission of the $k$-jet and polynomial truncation operator $J_{k}$ in the arguments below.

The algebraic conditions. In the case $u=\infty$ and $j=0$, the following algebraic condition $A C_{k, j}(f)$ is equivalent to that given by Mather for the contact $k$ determinacy of $f$.

Algebraic condition $A C_{k, j}(f)$. For each homogeneous polynomial map of order $k, h(x)=h\left(x_{1}, \ldots, x_{n}\right)=\sum_{\alpha,|\alpha|=k} h_{\alpha} x^{\alpha}$ with $R^{m}$-valued coefficients $h_{\alpha}$ there is an n-vector $W(x)=\left(W_{1}(x), \ldots, W_{n}(x)\right)$ and $a m \times m$ matrix $A(x)=$ $\left[A_{i j}(x)\right]_{1 \leq i, j \leq m}$, both with polynomial components such that

$$
h(x)=J_{k}\left[\left\{D_{x} p(x)\right\} \cdot W(x)+A(x) \cdot f(x)\right],
$$

$J_{j} W(x)=0$ and $J_{j} A(x)=0$.

In the above condition, the $k$-jet $p(x)$ may be replaced by the function $f(x)$ when $f$ is at least $C^{k+1}$. (If $f(x)$ is only $C^{k}$, then $J_{k} D f(x)$ is undefined.) The condition $A C_{k, j}(f)$ is equivalent to $A C_{k, j}(p)$.

The requirements $J_{j} W(x)=0$ and $J_{j} A(x)=0$ entail that all the polynomial components of $W(x)$ and $A(x)$ are $O\left(|x|^{j+1}\right)$ and hence $j$-flat.

The corresponding algebraic condition $A R_{k, j}(f)$ for right determinacy is obtained by taking the matrix-valued polynomial $A(x)$ to be the $m \times m$ zero matrix. In this case, due to the presence of good and bad dimensions, the condition $A R_{k, j}(f)$ is of interest only when (a) the range space $R^{m}$ has dimension $m=1$ or (b) the map $f$ is locally a submersion about the origin $x=0$. In any appearance of $A R_{k, j}(f)$ below, we assume $m=1$.

The implications

$$
A C_{k, j}(f) \Longrightarrow A C_{k+1, j+1}(f) \text { and } A R_{k, j}(f) \Longrightarrow A R_{k+1, j+1}(f)
$$

hold when $f$ is at $C^{k+1}$. In accordance with the use of the phrase Siersma shadow property in Poston and Stewart [1978], these implications are herein called the Siersma shadow properties. For sufficiently differentiable maps $f$ these Siersma shadow properties permit the nonnegative integers $k$ and $j$ to both be incremented by one. When $j>0$ some of the arguments below simplify.

For each positive number $a$, let $B_{a}\left(R^{n}\right)$ denote the ball of radius $a$ centered at the origin in $R^{n}$. Given positive integers $k$ and $u$ with $k \leq u<\infty$, 
let $C^{u}\left(B_{a}\left(R^{n}\right), R^{m}\right)$ be the Banach space of $C^{u}$ maps $h: B_{a}\left(R^{n}\right) \rightarrow R^{m}$. Further, let $\left(1-J_{k-1}\right) C^{u}\left(B_{a}\left(R^{n}\right), R^{m}\right)$ be the closed subspace consisting of those maps $h$ with vanishing $(k-1)$-jet $J_{k-1} h=0$. This subspace is the range of the projection operator $\left(1-J_{k-1}\right)$ when this operator acts on the space $C^{u}\left(B_{a}\left(R^{n}\right), R^{m}\right)$.

Theorem 3.1. On contact determinacy. Let $0 \leq j<k \leq u<\infty$. Set $r=u-k$. Assume $f$ is a $C^{u}, R^{m}$-valued, map defined on an open neighbourhood of the origin $x=0$ in $R^{n}$. Assume $f$ satisfies the algebraic condition $A C_{k, j}(f)$. Then for $a>0$ and $c>0$ sufficiently small, there are $C^{r}$ maps

$$
\left(A_{\alpha}(x, h), Y_{\alpha}(x, h)\right): B_{a}\left(R^{n}\right) \times\left(1-J_{k}\right) C^{u}\left(B_{a}\left(R^{n}\right), R^{m}\right) \rightarrow R^{m \times m} \times R^{n},
$$

vanishing whenever $h=0$ such that the $m \times m$ matrix-valued map

$$
A(x, h)=I_{m}+\sum_{|\alpha|=j+1} A_{\alpha}(x, h) x^{\alpha}=I_{m}+O\left(|x|^{j+1}\right)
$$

and the $R^{n}$-valued map

$$
y(x, h)=x+\sum_{|\alpha|=j+1} Y_{\alpha}(x, h) x^{\alpha}=x+O\left(|x|^{j+1}\right)
$$

are both $C^{r}$ maps which satisfy the following properties whenever $\|x\| \leq a$ and $\|h\|_{C^{k}} \leq c$.

I. $f(x)+h(x)=A(x, h) \cdot f(y(x, h))$,

II. $A(0, h)$ is nonsingular,

III. $y(x, 0)=x$,

IV. $A(x, 0)=I_{m}$ and

V. the partial $x$-Fréchet derivative represented by the Jacobian matrix $D_{x} y(0, h)$ exists and is nonsingular.

The full proof of Theorem 3.1 is given in $\S 6$ following some preliminaries in $\S \S 4$ and 5 . Most of the remaining assertions in this section are consequences or special cases of Theorem 3.1.

Differentiability notes.

(1) Observe the presence of the $C^{k}$ norm in the conclusion. The proof below of Theorem 3.1 shows the maps in its conclusion have a domain independent of $u$ when $u \geq k$. The functions in the conclusions are defined and continuous on a neighbourhood of the origin in the product Banach space $R^{n} \times\left(1-J_{k-1}\right) C^{k}\left(B_{a}\left(R^{n}\right), R^{m}\right)$. The latter is equipped with the usual product norm. The restriction of the maps to the intersection of the above domain with the product Banach spaces $R^{n} \times\left(1-J_{k-1}\right) C^{u}\left(B_{a}\left(R^{n}\right), R^{m}\right)$ yields $C^{r}=C^{u-k}$ maps when $u \geq k$. In the latter spaces, with respect to a product norm based on those of $R^{n}$ and $C^{u}\left(B_{a}\left(R^{n}\right), R^{m}\right)$, the domain of the restricted mappings is a neighbourhood of their common origin. Finally, if the map $f$ in Theorem 3.1 is a polynomial, for instance a $k$-jet, or an analytic function, then for $x$ fixed in 
the ball $\|x\| \leq a$, the maps in the conclusion and all their partial $x$-derivatives of order $\leq r=u-k$ determine analytic $R^{n}$ - and $R^{m \times m}$-valued functions of $h$ in the Banach space $\left(1-J_{k-1}\right) C^{u}\left(B_{a}\left(R^{n}\right), R^{m}\right)$. The text Berger [1977] gives a brief description of analytic functions on Banach spaces.

(2) In the event $u=\infty$ or $u=\omega$, we may regard the function space $C^{u}\left(B_{a}\left(R^{n}\right), R^{m}\right)$ and its subspace $\left(1-J_{k}\right) C^{u}\left(B_{a}\left(R^{n}\right), R^{m}\right)$ as Fréchet spaces equipped with the $C^{q}$ norms where $q \geq 0$. In this case for $h$ fixed, the maps in the conclusion are $C^{\infty}$ functions on the ball $\|x\| \leq a$ in $R^{n}$. Description of the further differentiability properties in this Fréchet space context is omitted because the lack of an appropriate terminology.

(3) If $u=\omega$ and $R$ is the complex number field, the associated spaces and subspaces of $C^{\omega}$ maps on the ball (or polydisc) $\|x\| \leq a$ equipped with the $C^{k}$ norm, form complex Banach spaces. The elements of these spaces are required say to be continuous on the interior of the ball or polydisc, and to be continuous upto the boundary of this domain together with their partial derivatives of order $k$ or less. Whence the $C^{k}$ norm is finite.

At the cost of decreasing the radius $a$ of the ball $\|x\| \leq a$, the Cauchy formula for functions of several complex variables permits the $C^{0}$, that is the sup-norm, to be employed instead. In either case, that is with either the $C^{k}$ norm or the sup-norm the conclusions of Theorem 3.1 hold as stated when $a>0$ and $c>0$ are sufficiently small.

(4) The case where $u=\omega$ and $R$ is the real number field by a complexification argument can be regarded as a special case of the situation in (3).

(5) An alternative in the analytic case $u=\omega$ which works whenever $R$ is the field of real or complex, and which even holds more generally when $R$ is a Banach algebra with a unit 1 , is to consider power series in $n$-variables $x=\left(x_{1}, \ldots, x_{n}\right)$ in the product Banach space $R^{n}$ which converge absolutely on a sufficiently small ball $\|x\| \leq a$. In particular, for Theorem 3.1 to hold as stated with $u=\omega$, take $C^{\omega}\left(B_{a}\left(R^{n}\right), R^{m}\right)$ to be the space of power series $h(x)=\sum_{\alpha \geq 0} h_{\alpha} \cdot x^{\alpha}$ with coefficients $h_{\alpha}$ in $R^{m}$ such that the weighted norm

$$
\|h\| \stackrel{\text { def }}{=} \sum_{\alpha \geq 0}\left\|h_{\alpha}\right\|_{R^{m}} \cdot a^{|\alpha|}
$$

is finite. The sum here is over all multi-indices $\alpha \geq 0$. These power series for $h(x)$ are absolutely convergent on the ball $\|x\| \leq a$. The coefficients $h_{\alpha}=\frac{1}{(\alpha) !} \partial^{\alpha} h(0)$ can be identified with the partial $\alpha$-(Fréchet) derivative of $h(x)$ in the Banach algebra case.

In the Banach algebra case, the power series $h(x)$ provide what I will call Lorch-analytic functions of several Banach algebra variables. The text Hille and Philips [1957] defines Lorch-analytic functions of a single variable. The definition can be generalized in an obvious manner to functions of a single or several variables in real or complex Banach algebras. 
With the weighted norm above, the two spaces $C^{\omega}\left(B_{a}\left(R^{n}\right), R\right)$ and $\left(1-J_{k-1}\right) C^{\omega}\left(B_{a}\left(R^{n}\right), R\right)$ of $R$-valued power series are Banach algebras.

In the Banach algebra case, the maps in the conclusions of Theorem 3.1 will be analytic functions, albeit not Lorch-analytic functions of the point

$$
(x, h)=\left(x_{1}, \ldots, x_{n}, h_{1}, \ldots, h_{m}\right)
$$

in the product Banach space $R^{n} \times\left(1-J_{k-1}\right) C^{\omega}\left(B_{a}\left(r^{n}\right), R^{m}\right)$. For fixed $h$, the maps will have power series expansion in $x \in R^{m}$ which converge absolutely say on the ball $\|x\| \leq a$.

The perturbation arguments, the polynomial approximations, and the polynomial representation of analytic maps below all hold for Lorch-analytic maps $f(x)$ given by power series in several Banach algebra variables which are absolutely convergent on a neighbourhood of the origin in $R^{n}$. The extension indicated here of $k$-determinacy results to functions of Banach algebra variables is thus feasible. But while Banach algebras abound, the utility of the indicated extension is at present unknown to me.

Sufficiency of $k$-jets. In the Theorem 3.1, the value of either $a$ or $c$ may be diminished without decreasing the other. Now $f(x)=J_{k} f(x)+h(x)$ where the $k$-jet of $h$ vanishes. So for $a>0$ sufficiently small, the $C^{k}$ norm of $h(x)$ on the ball $\|x\| \leq a$ is less than $c$. This implies $f$ is $j$-flat, $C^{r}$, contact equivalent to its $k$-jet $p(x)=J_{k} f(x)$.

Loss of fewer derivatives. In Theorem 3.1, there is a loss of $k$ derivatives in the construction of the change of variables and/or required matrix-valued maps when $u$ is finite. This loss stems from the polynomial representation of $C^{u}$ based on Taylor's formula in $\S 5$. Theorem 3.2 below restricts this loss to the origin $x=0$, and ensures the loss there is of at most $k-j-1$ derivatives. This result in the case of the Morse lemma was first given in the paper Ang and Vũ-Trong-Tuân [1979].

Theorem 3.2. On loss of fewer derivatives. Let $0 \leq j<k \leq u<\infty$. Let $a>0$ and $c>0$ be as in Theorem 3.1. Let $r=u-k+j+1 \geq u-k$. Assume $f$ is $a C^{u+k}, R^{m}$-valued, map defined on an open neighbourhood of the ball $B_{a}\left(R^{n}\right)$ in $R^{n}$. Further assume $f$ satisfies the algebraic condition $A C_{k, j}(f)$. Let $h$ be a map in the space $\left(1-J_{k}\right) C^{u}\left(B_{a}\left(R^{n}\right), R^{m}\right)$. Let $s$ be a real variable. Then for $b>0$ sufficiently small, there are $C^{u-k}$ maps

$$
\left(A_{\alpha}(x, s), Y_{\alpha}(x, s)\right): B_{a}\left(R^{n}\right) \times R \rightarrow R^{m \times m} \times R^{n},
$$

which are $C^{u}$ functions of their arguments when $x \neq 0$, which vanish at $s=0$, and are such that the $m \times m$ matrix-valued map

$$
A(x, s) \stackrel{\text { def }}{=} I_{m}+\sum_{|\alpha|=j+1} A_{\alpha}(x, s) x^{\alpha}=I_{m}+O\left(|x|^{j+1}\right)
$$


and the $R^{n}$-valued map

$$
y(x, s) \stackrel{\text { def }}{=} x+\sum_{|\alpha|=j+1} Y_{\alpha}(x, s) x^{\alpha}=x+O\left(|x|^{j+1}\right)
$$

are both $C^{r}$ functions, $C^{u}$ when $x \neq 0$, satisfying the following properties whenever $\|x\| \leq a, 0 \leq s \leq 1$, and $\|h\|_{C^{u}} \leq b$.

I. $f(x)+s \cdot h(x)=A(x, s) \cdot f(y(x, s))$,

II. $A(0, s)$ is nonsingular,

III. $y(x, 0)=x$,

IV. $A(x, 0)=I_{m}$, and

V. the partial $x$-Fréchet derivative represented by the Jacobian $D_{x} y(0, s)$ exists and is nonsingular.

Note the presence of the $C^{u}$ norm in the conclusion. Also note the hypotheses hold for the $k$-jet $p(x)=J_{k}[f(x)]$ of any function $f(x)$ satisfying the algebraic conditions $A C_{k, j}(f)$.

In the following proof $\left(J_{k}-J_{k-1}\right) R^{m}[x]$ stands for the space of homogeneous polynomials of degree and order $k$ with values in $R^{m}$.

Proof. Let $z(x)$ denote an homogeneous polynomial in the finite-dimensional subspace $\left(J_{k}-J_{k-1}\right) R^{m}[x]$ of $\left(1-J_{k}\right) C^{u+k}\left(B_{a}\left(R^{n}\right), R^{m}\right)$. Let $a$ and $c$ be as in the conclusion of Theorem 3.1. Then for $\|z\|<c$,

$$
f(x)+z(x)=A(x, z) \cdot f(y(x, z))
$$

where the vector-valued map $y(x, z)=x+O\left(|x|^{j+1}\right)$ and the $m \times m$ matrix $A(x, z)=I_{m}+O\left(|x|^{j+1}\right)$ have a $C^{u}$ dependence on $x$ and the coefficients of the polynomial $z(x)$. Note the employment here of the assumption that $f$ is $C^{u+k}$ rather than $C^{u}$.

Theorem 1.2.1 in Ang and Vũ-Trong-Tuân [1979] is expressed in terms of Fréchet derivatives and multi-linear maps in a coordinate-free form. It implies the following in terms of coordinates. Let $h(x)=O\left(|x|^{k}\right)$ be an element of the subspace $\left(1-J_{k-1}\right) C^{u}\left(B_{a}\left(R^{n}\right), R^{m}\right)$. Then by Theorem 1.2.1

$$
h(x)=\sum_{|\alpha|=k} h_{\alpha}(x) \cdot x^{\alpha}
$$

where the coefficients $h_{\alpha}(x)$ are $C^{u-k}$ functions on the ball $B_{a}\left(R^{n}\right)$, which are also $C^{u}$ when $x \neq 0$, and which further satisfy the limit conditions

$$
0=\lim _{x \rightarrow 0}\|x\|^{|\beta|-k} \partial^{\beta} h_{\alpha}(x)
$$

where $k \leq|\beta| \leq u$. Here $\beta=\left(\beta_{1}, \ldots, \beta_{n}\right)$ is a multi-index with nonnegative integer components.

For $t \in B_{a}\left(R^{n}\right)$, we next define a $t$-dependent polynomial $z(t)(x)$. Put $z(t)(x)=\sum_{|\alpha|=k} h_{\alpha}(t) \cdot x^{\alpha}$. Then $z(t)(x)$ a homogeneous polynomial of order 
$k$ in $x$ with coefficients depending continuously on $t$ in the ball $B_{a}\left(R^{n}\right)$. The parameter-dependent family of polynomials $z(t)(x)$ in $x$ gives a representation of the map $h$ that reproduces $h(x)$ or $h(t)$ on the substitution $x=t$. Another parameter-dependent polynomial representation of polynomials is introduced in $\S 5$.

The division-based representation for the remainder term in Taylor's formula from Ang and Vũ-Trong-Tuân [1979] implies the coefficients of the polynomial $z(t)(x)$ are elements of the Banach space $C^{0}\left(B_{a}\left(R^{n}\right), R^{m}\right)$. As functions valued in the last Banach space of $C^{0}$ maps, the coefficients $h_{\alpha}(\cdot)$ are given (by construction) by continuous linear functions of $h$ in the Banach space $\left(1-J_{k-1}\right) C^{u}\left(B_{a}\left(R^{n}\right), R^{m}\right)$. Hence when the $C^{u}$ norm of $h$ sufficiently small, say $\|h\|_{C^{u}}<b$ for some positive $b$, then for each fixed $t$ in $B_{a}\left(R^{n}\right)$, the $C^{k}$ norm of the associated polynomial map $z(t)(\cdot)$ in $\left(1-J_{k-1}\right) C^{u}\left(B_{a}\left(R^{n}\right), R^{m}\right)$ satisfies $\|z(t)(\cdot)\|_{C^{k}}<c$.

The foregoing entails $f(x)+s \cdot z(t)(x)=A(x, s \cdot z(t)) \cdot f(y(x, s \cdot z(t)))$ for $\|x\| \leq a$ and $0 \leq s \leq 1$. The substitution $x=t$ in conjunction with the above map reproducing property yields

$$
f(t)+s \cdot h(t)=A(t, s \cdot z(t)) \cdot f(y(t, s \cdot z(t))) .
$$

With a small change in notation, including the replacement of $x$ by $t$, the last equality yields the change of variables and matrix-valued map required in the conclusion.

The required $C^{u}$ dependence for $x \neq 0$ follows easily from the chain-rule.

The required $C^{r}$ dependence where $r=u-k+j+1$ follows from the iterated chain-rule and the partial converse to Taylor's formula given in Theorem 1.2.2 of Ang and Vũ-Trong-Tuân [1979]. The satisfaction of the hypotheses of Theorem 1.2.2 follow from the above limit conditions.

Right determinacy. Satisfaction of right rather than contact determinacy conditions above produces the next theorem.

Theorem 3.3. On right determinacy. In Theorem 3.1 and in Theorem 3.2 assume $m=1$. Further assume the right determinacy condition $A R_{k, j}(f)$. Then the conclusions of both Theorem 3.1 and Theorem 3.2 hold with the matrix $A(x, h)=1 \in R$.

Necessity of the algebraic conditions. A map $f: R^{n} \rightarrow R^{m}$ is here said to be differentiably, $j$-flat, $C^{r}$ contact $k$-determined at the origin in the class of $C^{u}$ perturbations $h(x)=O\left(|x|^{k+1}\right)$ iff for each such $C^{u}$ perturbation $h(x)$

(1) there is a $C^{r}$ map $y(x, s)$ on the space $R^{n} \times R$ with $y(x)=x+$ $O\left(|x|^{j+1}\right)$ for $s$ fixed; and

(2) there is a $m \times m$ matrix-valued $C^{r}$ function $A(x, s)$ with $A(x, s)=$ $I_{m}+O\left(|x|^{j+1}\right)$ and $A(0, s)$ nonsingular for $s$ fixed

such that $A(x, s) \cdot f(y(x, s))=f(x)+s \cdot h(x)$ on some neighbourhood of the origin in $R^{n} \times R$. The corresponding definition of differentiably, $j$-flat, 
$C^{r}$ right $k$-determinacy is obtained by taking $A(x, s)=I_{m}$. Converses to the foregoing $C^{r}$ determinacy Theorems 3.1, 3,2 and 3.3 when $r>k$ are implied by the following.

Theorem 3.4. On necessity of algebraic conditions. Let $0 \leq j<k<r$ and $k<u$. Further, assume the $C^{u}$ map $f: R^{n} \rightarrow R^{m}$ is j-flat, differentiably $C^{r}$ contact (resp., right) $k$-determined at the origin in the class of $C^{u}$ perturbations $h(x)=O\left(|x|^{k+1}\right)$. Then the algebraic condition $A C_{k, j}(f)\left[\right.$ resp., $\left.A R_{k, j}(f)\right]$ holds.

Proof. Consider the following tangent space argument. Let $h(x)=\sum_{|\alpha|=k} h_{\alpha}$. $x^{\alpha}$ be an $R^{m}$-valued homogeneous polynomial in $x$. Then for $s$ real and sufficiently small,

$$
f(x)+s \cdot h(x)=\left[I_{m}+a(x, s)\right] \cdot f(x+w(x, s))
$$

where $a(x, s)=O\left(|x|^{j+1}\right)$ and $w(a, s)=O\left(|x|^{j+1}\right)$ are both $C^{r}$ maps vanishing when $s=0$. Differentiation with respect to the parameter $s$ at $s=0$ yields

$$
h(x)=\left.\frac{\partial}{\partial s}\right|_{s=0} a(x, s) \cdot f(x)+\left.D_{x}[f(x)] \cdot \frac{\partial}{\partial s}\right|_{s=0} w(x, s) .
$$

Apply the $J_{k}$ operator to this last equality. Then set

$$
A(x)=\left.J_{k} \frac{\partial}{\partial s}\right|_{s=0} a(x, s) \quad \text { and } \quad W(x)=\left.J_{k} \frac{\partial}{\partial s}\right|_{s=0} w(x, s) .
$$

Condition $A C_{k, j}(f)$ follows immediately since $\mathrm{h}$ is arbitrary. The necessity of $A R_{k, j}(f)$ is shown similarly simply by taking $a(x, s)=1$.

Robustness. For a given $C^{u}$ function $f: R^{n} \rightarrow R^{m}$, say the algebraic condition $A C_{k, j}(f)$ [resp., $A R_{k, j}(f)$ ] is robust with respect to kth order perturbations iff for each $C^{u}$ (or polynomial) map $h: R^{n} \rightarrow R^{m}$ with $(k-1)$-jet $J_{k-1} h=0$, the algebraic condition $A C_{k, j}(f+h)$ [resp., $A R_{k, j}(f+h)$ ] also holds. The algebraic conditions $A C_{k, j}(f)$ and $A R_{k, j}(f)$ are easily seen to be robust whenever $j>$ 0 . Theorem 3.5 follows or extends the corresponding assertions in Wasserman [1974].

Theorem 3.5. On $(k-1)$-determinacy. Assume $0 \leq j<k$ and suppose $k \leq$ $u \leq \infty$ or $u=\omega$. Set $r=u-k+j+1$. Assume $f$ is a $C^{u}, R^{m}$-valued, map defined on an open neighbourhood of the origin $x=0$ in $R^{n}$. Finally, the algebraic condition $A C_{k, j}(f)\left[\right.$ resp., $\left.A R_{k, j}(f)\right]$ is robust. Then $f$ and all of its $C^{u}$ perturbations $f+g$ with $g(x)=O\left(|x|^{k-1}\right)$ are differentiably, j-flat, $C^{r}$ contact (resp., right) $(k-1)$-determined in the class of $C^{u}$ perturbations $f+h: R^{n} \rightarrow R^{m}$ where $h(x)=O\left(|x|^{k-1}\right)$ is $C^{u}$.

The converse to Theorem 3.5 holds when $r>k$. The proof is omitted. It is similar to that of Theorem 3.4. 
Proof. Equip the vector space of $k$ th-order $C^{u}$ maps $h: R^{n} \rightarrow R^{m}$ with a topology induced by the $k$-jet (semi)norm $\left\|J_{k} h\right\|$. By Theorem 3.2, the collection of maps $h$ in this vector space for which $f+h$ is $j$-flat, $C^{r}$ contact equivalent to $f$ is easily seen to be nonvoid and open. Its complement is also open. The claim follows immediately by the connectedness of topological vector spaces.

Remark A. The survey article Wall [1981] presents an assertion Theorem 8.1 on the right and contact $C^{r}$ determinacy of $C^{\infty}$ and sufficiently differentiable maps, but its proof is flawed. Let $r>1$ denote an integer. The proof of Theorem 8.1 assumes a map of the form $\psi(x)=O\left(|x|^{r+1}\right)$ which is $C^{\infty}$ for $x \neq 0$ to be $C^{r}$. The map $\psi(x)=x^{r+1} \sin \left(x^{-(1+r)}\right)=O\left(|x|^{r+1}\right)$ gives a counterexample. It is $C^{\infty}$ except at the origin. It is once differentiable everywhere, but it is not continously differentiable as $\psi^{\prime}(x)=O\left(\frac{1}{|x|}\right)$ when $x \rightarrow 0$. Hence $\psi(x)$ is not $C^{1}$ nor $C^{r}$. Theorem 8.1 and the error in its proof were brought to my attention by the referee.

Remark B. With the elliptic-like coercive hypotheses for $C^{r}$, $j$-flat, contact $k$-determinacy as in Lefebvre and Pourprix [1984], the construction of the required changes variables and/or matrix transformation required in right and contact determinacy arguments involves the integration of $C^{r}$ systems of ordinary differential equations. The construction results in families of changes of variables and/or matrix transformations with a $C^{r}$ dependence on a small parameter. So the required maps have a $C^{r}$ dependence on the small parameter. Now for $r>k$ necessity result Theorem 3.4 applies and hence the algebraic condition $A C_{k, j}(f)$ holds. If $0 \leq r \leq k$, the result, Theorem 3.4 does not apply. There are $C^{\infty}$ functions which satisfy the elliptic-condition of Kuo [1979a] and others, but not the algebraic conditions of Mather.

Remark C. Conjecture. Theorem 3.1 may also holds with $r=u-k+j+$ 1 instead of $r=u-k$. This could follow from the converse to Taylor's formula and a generalization of Theorem 1.2.2 in Ang and Vũ-Trong-Tuân [1979]. (Applications of the converse to Taylor's formula are given in Abraham and Robbin [1967]. A short and neat proof of the converse is given in Nelson [1969].)

Remark D. The ideas for the proof of a $k$-determinacy result stem from reading the introductory text Poston and Stewart [1978]. During this introduction, I hoped the demonstration of right determinacy and versal unfolding would follow the model provided by the generalised Morse lemma of Hormander [1971] or rather its demonstration, as presented in the lecture notes Nirenberg [19731974]. The latter is in part reminiscent of the usually formal two-timing or two-variable method described say in Nayfeh [1973]. 
Remark E. The versal unfolding of analytic maps with respect to contact and right equivalence relations can also be obtained from the the ordinary implicit function theorem in an infinite-dimensional perturbation argument. This derivation appears to be new. So details may be given in a forthcoming article.

Remark $\mathrm{F}$. The blowing up method from algebraic geometry, along with some nondegeneracy assumptions on the blown-up equations are employed in Bucher, Marsden and Schecter [1983] to obtain determinacy results for $k$ th-order maps. The differentiability assertions in the article are based on the paper Ang and VũTrong-Tuân [1979].

\section{Polynomial APPROXIMATIONS to $k$ TH-ORDER}

Notation. Let $x=\left(x_{1}, \ldots, x_{n}\right)$ denote a vector of $n$ variables or indeterminates. For each finite-dimensional vector space $V$, let $V[x]=V\left[x_{1}, \ldots, x_{n}\right]$ be the collection of polynomials $v(x)=\sum_{\alpha} v_{\alpha} x^{\alpha}$ in the variables $\left(x_{1}, \ldots, x_{n}\right)$ with coefficients $v_{\alpha}$ in the vector space $V$. Only finitely many coefficients are allowed to be nonzero. Let $R^{m \times m}$ denote the set of matrices with $m$ rows and $m$ columns. The two cases $V=R^{n}$ and $V=R^{m \times m}$ result in spaces of vector-valued polynomials $R^{n}[x]$ and matrix-valued polynomials $R^{m \times m}[x]$, respectively.

The jet operators $J_{k}$ and $J_{j}$ act on $V[x]$ as truncation and projection operators. The finite-dimensional vector space of $V$-valued polynomials in $\left(x_{1}, \ldots, x_{n}\right)$ of degree at most $k$ is denoted by $J_{k} V[x]$. It consists of the range of the projection operator $J_{k}: V[x] \rightarrow V[x]$. This space is finite dimensional. It can be equipped with a norm. Any norm will suffice.

The operator $J_{k}$ acts as the identity on its range $J_{k} V[x]$. The operator $\left(J_{k}-J_{j}\right)$ acts as a projection operator on $J_{k} V[x]$. The subspace $\left(J_{k}-J_{j}\right) V[x]=$ $\left(1-J_{j}\right) J_{k} V[x]$ in $J_{k} V[x]$ consists of $j$-flat, $V$-valued, polynomials $v(x)=$ $\sum_{j<|\alpha| \leq k} v_{\alpha} x^{\alpha}=O\left(|x|^{j+1}\right)$ of order $j+1$ and degree at most $k$. The subspace $\left(J_{k}-J_{k-1}\right) V[x]$ is the collection of $V$-valued polynomials $h(x)=\sum_{|\alpha|=k} h_{\alpha} x^{\alpha}$ which are homogeneous and of order $k$ with coefficients $h_{\alpha}$ in the space $V$. The subspace $\left(J_{k}-J_{0}\right) V[x]$ consists of polynomials which are $O(|x|)$ and which therefore vanish at the origin.

The addition of the $m \times m$ identity matrix $I_{m}$ to each element of the subspace $\left(J_{k}-J_{j}\right) R^{m \times m}[x]$ yields the linear submanifold $I_{m}+\left(J_{k}-J_{j}\right) R^{m \times m}[x]$ in the polynomial space $J_{k} R^{m \times m}[x]$. The addition of the identity map $\operatorname{Id}(x)=x$ to each element of the subspace $\left(J_{k}-J_{j}\right) R^{n}[x]$ yields a linear submanifold $x+\left(J_{k}-J_{j}\right) R^{n}[x]$ in the polynomial space $J_{k} R^{n}[x]$.

The approximations. Lemmas 4.1 and 4.2 provide the approximations needed for our contact and right determinacy arguments. 
Lemma 4.1. $k$ th-order contact determinacy. Suppose the $C^{k}$ map $f: R^{n} \rightarrow R^{m}$ satisfies the algebraic condition $A C_{k, j}(f)$. Then there are analytic polynomialvalued functions

$$
(\Phi(h), \Psi(h)):\left(J_{k}-J_{k-1}\right) R^{m}[x] \rightarrow\left(J_{k}-J_{j}\right) R^{n}[x] \times\left(J_{k}-J_{j}\right) R^{m \times m}[x]
$$

and an $\varepsilon_{j}>0$ and $\leq \infty$ such that

$$
J_{k} f(x)+h(x)=J_{k}\left[I_{m}+\Psi(h)(x)\right] \cdot f(x+\Phi(h)(x))
$$

$\operatorname{det}\left(I_{m}+\Psi(h)(0)\right) \neq 0$ and $\operatorname{det}\left(D_{x}(x+\Phi(h)(0))\right) \neq 0$ whenever the norm $\|h\|$ of the homogeneous polynomial $h: R^{n} \rightarrow R^{m}$ in $\left(J_{k}-J_{k-1}\right) R^{m}[x]$ satisfies $\|h\|<\varepsilon_{j}$. Moreover, $(\Phi(h), \Psi(h))=(0,0)$ when $h=0$. Moreover, if $j>0$ then the foregoing holds with $\varepsilon_{j}=\infty$.

Lemma 4.2. $k$ th-order right determinacy. Suppose a $C^{k} \operatorname{map} f: R^{n} \rightarrow R$ satisfies the algebraic condition $A R_{k, j}(f)$. Then Lemma 4.1 holds with $\Psi(h)(x)=0$ identically, and $m=1$.

The ranges of the maps in Lemma 4.1 and Lemma 4.2 form submanifolds in finite-dimensionsal spaces or product spaces of polynomials. The construction of the required submanifolds follows below from the integration of systems of ordinary differential equations. An alternative method is to employ an exponential maps on Lie groups of polynomial maps equipped with a truncated compostion operation. For a discussion of the Lie group aspects of Lemmas 4.1 and 4.2, see the appendix to the present section and see Martinet [1982] or Selby [1983]. The Lie group aspects of the calculations are superflous here but they are included here as a link to the Lie group perspective taken in the literature.

\section{Proof of Lemma 4.1.}

Step A: Linear polynomial-valued functions.

The finite-dimensional space $\left(J_{k}-J_{k-1}\right) R^{m}[x]$ of $R^{m}$-valued, homogeneous polynomials of order $k$ by hypothesis lies in the range of the linear map $L$ defined by

$$
L[A(\cdot), W(\cdot)](x) \stackrel{\text { def }}{=} J_{k}\left[A(x) \cdot p(x)+\left(D_{x} p(x)\right) \cdot W(x)\right] .
$$

Here $A(x)$ and $W(x)$ are polynomials of order $j+1$ in $\left(J_{k}-J_{j}\right) R^{m \times m}[x]$ and in $\left(J_{k}-J_{j}\right) R^{n}[x]$ respectively.

Let $e_{1}, \ldots, e_{m}$ denote a basis of $R^{m}$. Then the monomials $x^{\alpha} \cdot e_{i}$ where $|\alpha|=k$ and $1 \leq i \leq m$ form a basis of the space $\left(J_{k}-J_{k-1}\right) R^{m}[x]$. Let $h(x)=\sum_{1 \leq i \leq m,|\alpha|=k} h_{i, \alpha} \cdot x^{\alpha} \cdot e_{i}$. By hypothesis, each

$$
x^{\alpha} \cdot e_{i}=J_{k}\left[A_{i, \alpha}(x) \cdot p(x)+\left(D_{x} p(x)\right) \cdot W_{i, \alpha}(x)\right]
$$

for some polynomials $A_{i, \alpha}(x)=O\left(|x|^{j+1}\right)$ and $W_{i, \alpha}(x)=O\left(|x|^{j+1}\right)$. Now put

$$
a(h)(x)=\sum_{1 \leq i \leq m,|\alpha|=k} h_{i, \alpha} \cdot A_{i, \alpha}(x)=O\left(|x|^{j+1}\right)
$$


and

$$
w(h)(x)=\sum_{1 \leq i \leq m,|\alpha|=k} h_{i, \alpha} \cdot W_{i, \alpha}(x)=O\left(|x|^{j+1}\right) .
$$

Then by linearity, $L(a(h)(\cdot), w(h)(\cdot))(x)=h(x)$. The coefficients of the polynomials $a(h)(x)$ and $w(h)(x)$ are linear functions of those of $h(x)$. These polynomial-valued maps $(a(\cdot)(x), w(\cdot)(x))$ together define a right inverse of the linear operator $L$.

Step B: Definition of the function $\Phi(h)(x)$.

For a real parameter $t$, which we may refer to as time, we define in this step a curve $Y(t)(x)$ in the linear submanifold $x+\left(J_{k}-J_{j}\right) R^{n}[x]$ of $\left(J_{k}-J_{0}\right) R^{n}[x]$ so that $Y(0)(x)=x$ and $Y(1)(x)=x+\Phi(h)(x)$. The construction of $Y(t)(x)$ follows.

Consider the initial value problem given by the nonlinear first-order system of ordinary differential equations

(ODE1)

$$
\frac{d Y(x)}{d t}=J_{k}\{w(h)(Y(x))\}
$$

together with the initial condition $Y(0)(x)=x$. This single equation represents a first-order system for the coefficients of the polynomial $Y$. A few words follow about (ODE1). In (ODE1) the polynomial $Y(x)=Y(t)(x)$ replaces the variable $x$ in the $h$-dependent polynomial $w(h)(x)$. Next the operator $J_{k}$ truncates terms of order $k+1$ and greater. The resulting polynomial $J_{k}[w(h) \circ Y](x)$ is of degree $k$ in $x=\left(x_{1}, \ldots, x_{n}\right)$. In this polynomial, the coefficient of each monomial $x^{\alpha}$ is a polynomial expression itself in the coefficients of the polynomial $Y(x)$ and on the coefficients of the polynomial $w(h)(x)$. Recall here, the linear dependence of $w(h)(x)$ on the homogeneous polynomial $h$. Therefore, the equation (ODE1) represents a parameter-dependent, analytic, and nonlinear first-order system of ordinary differential equations for the coefficients of the $t$-dependent polynomial Y. The parameters are the coefficients of $h$.

Let $Y(x)$ belong to the linear submanifold $x+\left(J_{k}-J_{j}\right) R^{n}[x]$ of the space $\left(J_{k}-J_{0}\right) R^{n}[x]$. The latter space consists of polynomials $v(x)=O\left(|x|^{0+1}\right)$ of degree $k$ or less. Here $Y(x)=O\left(|x|+|x|^{j+1}\right)=O(|x|)$.

Now $w(h)(x)=O\left(|x|^{j+1}\right)$ implies the polynomial

$$
J_{k} w(h)(Y(x))=O\left(|Y(x)|^{j+1}\right)=O\left(|x|^{j+1}\right) .
$$

Therefore the polynomial $J_{k} w(h)(Y(x))$ lies in the tangent space $\left(J_{k}-J_{j}\right) R^{n}[x]$ of the linear manifold $x+\left(J_{k}-J_{j}\right) R^{n}[x]$. Equivalently, the coefficients of the monomial $x^{\alpha}$ on the right-hand side of the first-order system (ODE1) vanish whenever $0<|\alpha| \leq j$. So the linear submanifold $x+\left(J_{k}-J_{j}\right) R^{n}[x]$ is invariant under the flow of the above first-order equation.

Let $Y(t)(x)$ be the solution of (ODE1) determined by the initial condition $Y(0)(x)=x$. Then by the above considerations, $Y(t)(x)$ lies in the invariant 
linear manifold $x+\left(J_{k}-J_{j}\right) R^{n}[x]$. Thus, whenever it is defined, the polynomial $Y(t)(x)=x+O\left(|x|^{j+1}\right)$.

The exponential property of the curve $Y(t)(x)$ is a truncated, polynomial composition property

$$
Y(t+s)(x)=J_{k}[Y(t) \circ Y(s)(x)]
$$

which holds whenever both sides are defined. The exponential property follows from the next calculation. Suppose $Y(s)(x)$ is defined. Now define a $t$-dependent curve

$$
Z(t)(x)=J_{k}[Y(t) \circ Y(s)(x)]=J_{k}[Y(t)(Y(s)(x))] .
$$

With a prime indicating differentiation with respect to $t$,

$$
\begin{aligned}
Z^{\prime}(t)(x) & =J_{k}\left[Y^{\prime}(t)(Y(s)(x))\right]=J_{k}\left[\left\{J_{k} w \circ Y(t)(\cdot)\right\}(Y(s)(x))\right] \\
& =J_{k}[w(Y(t) \circ(Y(s)(x)))] .
\end{aligned}
$$

Hence $Z^{\prime}(t)=J_{k}[w(h)(Z(t)(x))]$. Now the curve $z(t)(x)=Y(t+s)(x)$ and the curve $Z(t)(x)$ satisfy the same ordinary differential equation (ODE1), and both initially pass through $Y(s)(x)$ at $t=0$. By uniqueness theory for initial value problems, the two curves coincide. Hence, the exponential property which can be written as $Z(t)=z(t)$ holds.

The above exponential property implies the curve $Y(t)(x)$ can be defined for all real $t$. (Proof: From uniqueness theory for analytic, ordinary differential equations, the solution curve $Y(t)(x)$ exists for $t$ sufficiently small, say for $t$ in an interval $[0, b]$ where $b>0$. From the exponential property, the solution curve $Y(t)(x)$ can be continued onto the interval $[b, 2 b]$ by setting $Y(b+t)(x)=J_{k} Y(t) \circ Y(b)(x)$ for $t$ in the interval $[0, b]$. The interval of definition $[0, b]$ of $Y(t)(x)$ can be doubled and then redoubled repeatedly until it contains all real $t \geq 0$. Existence for $t \leq 0$ follows similarly.)

By the theory of analytic ordinary differential equations, the solution curve $Y(t)(x)=x+\Phi_{0}(h, t)(x)$ for some analytic map $\Phi_{0}(h, t)(x)$ valued in the linear manifold $x+\left(J_{k}-J_{j}\right) R^{n}[x]$. The coefficients of the $(t, h)$-dependent polynomial $\Phi_{0}(h, t)(x)$ here are analytic functions of time $t$ and the coeffcients of the homogeneous polynomial $h$.

Linearity of the map $h \rightarrow w(h)(x)$ and in particular the property $w(s \cdot h)(x)=s \cdot w(h)(x)$ of $w(h)(x)$ for $s$ real implies the rescaling property $x+\Phi_{0}(h, s t)(x)=x+\Phi_{0}(s h, t)(x)$ since both sides satisfy the same initial conditions and the same rescaled version of equation (ODE1). Multiplication of the right-hand side of (ODE1) by the real factor $s$ provides the rescaled version.

Let

$$
\Phi(h)(x) \stackrel{\text { def }}{=} Y(1)(x)-x=\Phi_{0}(h, 1)(x) \in\left(J_{k}-J_{j}\right) R^{n}[x] .
$$

Then $\Phi(h)(x)=O\left(|x|^{j+1}\right)$ for each fixed $h$. 
Substep: On the nonsingularity of the Jacobian matrix $\left.D_{x}[x+\Phi(h)(x)]\right|_{x=0}$

If $j>0$ then $\left.D_{x}[x+\Phi(h)(x)]\right|_{x=0}=I_{n}=$ the $n \times n$ identity matrix since $\Phi(h)(x)=O\left(|x|^{j+1}\right)=O\left(|x|^{2}\right)$. In the case $j=0$ observe (ODE1) implies the linear cofficient $D_{x} Y(t)(0)$ in $Y(t)(x)=D_{x} Y(t)(0) \cdot x+O\left(|x|^{2}\right)$ satisfies

$$
\frac{d D_{x} Y(t)(0)}{d t}=D_{x} w(h)(Y(t)(0)) \cdot D_{x} Y(t)(0)=D_{x} w(h)(0) \cdot D_{x} Y(t)(0)
$$

The initial condition $Y(0)(x)=x$ now entails $D_{x} Y(t)(0)=\exp \left(t \cdot D_{x} w(h)(0)\right)$ is the exponential of a matrix. Thus the Jacobian matrix $\left.D_{x}[x+\Phi(h)(x)]\right|_{x=0}$ $=D_{x} Y(1)(0)$ is nonsingular.

Step C: Definition of the function $\Psi(h)(x)$.

For a real parameter $t$, which may again be referred to as time, we define in this step a curve $A(t)(x)$ in the linear submanifold $I_{m}+\left(J_{k}-J_{j}\right) R^{m \times m}[x]$ of $J_{k} R^{m \times m}[x]$.

Consider the initial value problem given by the first-order system of ordinary differential equations

$$
\frac{d A}{d t}(x)=J_{k}\{A(x) \cdot a(h)(Y(t)(x))\},
$$

together with the initial and the initial condition $A(0)(x)=I_{m}$ at $t=0$. Here the analytic map $Y(t)(x)=x+\Phi(h, t)(x)$ was defined in Step B. The coefficients of $Y(t)(x)=x+\Phi(h, t)(x)$ depend analytically on $t$ and those of the homogeneous polynomial $h$. The initial condition determines a polynomialvalued curve $A(t)(x)$ which passes through the $m \times m$ identity matrix $I_{m}$ at the initial time $t=0$.

The equation (ODE2) defines a linear nonautonomous first-order system for the coefficients of the polynomial $A(t)(x)$ with analytic dependence on time $t$ and the coefficients of the polynomial parameter $h(x)$. Therefore the solution curve $A(t)(x)$ in $J_{k} R^{m \times m}[x]$ exists for all time $t$ and has analytic dependence on time $t$ and the polynomial parameter $h$. Further from $a(h)(Y(t)(x))=O\left(|Y(t)(x)|^{j+1}\right)=O\left(|x|^{j+1}\right)$, the $j$-jet of the polynomialcurve $A(t)(x)$ is $t$-independent. To see this, note the right-hand side of the system (ODE2) is $O\left(|x|^{j+1}\right)$. Therefore $J_{j} A^{\prime}(t)(x)=0$. Integration yields $J_{j} A(t)(x)=J_{j} A(0)(x)=x$. Hence, the initial condition implies that $A(t)(x)=$ $I_{m}+\psi_{0}(h, t)(x)$ for some polynomial $\psi_{0}(h, t)(x)=O\left(|x|^{j+1}\right)$ with analytic dependence on $t$ and the coefficients of $h$. Let

$$
\Psi(h)(x) \stackrel{\text { def }}{=} A(1)(x)-I_{m}=\psi_{0}(1, h)(x)=O\left(|x|^{j+1}\right) .
$$

Substep: The nonsingularity of $\left.\left[I_{m}+\Psi(h)(x)\right]\right|_{x=0}$.

It is obvious $\left.\left[I_{m}+\Psi(h)(x)\right]\right|_{x=0}=I_{m}$ when $j>0$ since $\Psi(h)(x)=O\left(|x|^{j+1}\right)$ $=O\left(|x|^{2}\right)$. In the case $j=0$, observe (ODE2) implies

$$
\frac{d A}{d t}(0)=\left.\left[J_{k} A(x) \cdot a(h)(Y(t)(x))\right]\right|_{x=0}=A(0) \cdot a(h)(0) .
$$


Hence $A(t)(0)=\exp (t a(h)(0)))$ is a the exponential of a matrix. So $\left[I_{m}+\right.$ $\Phi(h)(0)]=[A(1)(0)]$ is nonsingular.

Step D: Conclusions when $j>0$.

Fix a homogeneous polynomial map $h(x)=O\left(|x|^{k-1}\right)$ with values in $R^{m}$. Let $Y(t)(x)$ and $A(t)(x)$ be the solutions of the initial value problems given above. Then

$$
\begin{aligned}
\frac{d}{d t} J_{k} & \{A(t)(x) \cdot f(Y(t)(x))\} \\
= & \frac{d}{d t} J_{k}\left\{A(t)(x) \cdot\left(J_{k} f\right)(Y(t)(x))\right\}, \quad \text { by iterated chain-rule, } \\
= & \frac{d}{d t} J_{k}\{A(t)(x) \cdot p(Y(t)(x))\}, \quad \text { since } p=J_{k} f, \\
= & J_{k}\left\{A^{\prime}(t)(x) \cdot p(Y(t)(x))+A(t)(x) \cdot\left(D_{x} p\right)(Y(t)(x)) \cdot Y^{\prime}(t)(x)\right\} \\
& \quad \text { by the product and chain-rules, } \\
= & J_{k}\left\{\left[J_{k} A(t)(x) a(h)(Y(t)(x))\right]\right\} \cdot p(Y(t)(x)), \quad \text { by Steps B and C, } \\
& \left.\quad+A(t)(x) \cdot\left(D_{x} p\right)(Y(t)(x)) \cdot\left[J_{k} w(h)(Y(t)(x))\right]\right\} \\
= & \left.J_{k}\left\{A(t)(x) \cdot[a(h)(\cdot)] p(\cdot)+\left(D_{x} p\right)(\cdot) w(h)(\cdot)\right] \circ Y(t)(x)\right\}, \\
& =J_{k}\{A(t)(x) \cdot[L(a(h)(\cdot), w(h)(\cdot))] \circ Y(t)(x)\}, \quad \text { by Step A, }, \\
& =J_{k}\{A(t)(x) \cdot[h] \circ Y(t)(x)\} \quad \text { as } h(x)=L(a(h)(\cdot), w(h)(\cdot))(x), \\
& \left.=J_{k}\left\{I_{m}+O\left(|x|^{j+1}\right)\right\} \cdot\left[h\left(x+O\left(|x|^{j+1}\right)\right)\right]\right\}, \quad \text { by Steps B and C, } \\
= & J_{k}\left\{\left[I_{m}+O\left(|x|^{2}\right)\right] \cdot\left[h\left(x+O\left(|x|^{2}\right)\right)\right]\right\}, \quad \text { as } j>0, \\
= & J_{k}\left\{I_{m} h(x)+O\left(|x|^{k+1}\right)\right\}, \quad \text { since } h(x)=O\left(|x|^{k}\right), \\
= & h(x), \quad \text { since } h(x)=O\left(|x|^{k}\right) .
\end{aligned}
$$

Observe the above equalities hold for $j \geq 0$ as well until the third to last equality where $j>0$ is needed. This observation is useful in the examination of the case $j=0$ in Step E below.

Integration with respect to $t$ in the interval $[0,1]$ yields

$$
\begin{aligned}
J_{k}\{A(1)(x) \cdot f(Y(1)(x))-f(x)\} & =\left.J_{k}\{A(t)(x) \cdot f(Y(t)(x))\}\right|_{t=0} ^{t=1} \\
& =\int_{0}^{1} \frac{d}{d t} J_{k}\{A(t)(x) \cdot f(Y(t)(x))\} d t \\
& =\int_{0}^{1} h(x) d t=h(x) .
\end{aligned}
$$

From this, the equalities $x+\Phi(h)(x)=Y(1)(x)$ and $I_{m}+\Psi(h)(x)=A(1)(x)$ together imply

$$
J_{k}\left\{\left[I_{m}+\Psi(h)(x)\right] \cdot f(x+\Phi(h)(x))\right\}=J_{k} f(x)+h(x)
$$

as required. The conclusions when $j>0$ have been established. 
Step E: Conclusion when $j=0$.

The following argument for the case $j=0$ involves a reparameterization of the polynomial-valued functions $(\Psi(h)(x), \Phi(h)(x))$ of $h$ in the space $\left(J_{k}-J_{k-1}\right) R^{m}[x]$. The argument $h$ is replaced by a map $z(h)(\cdot)$ of the polynomial space $\left(J_{k}-J_{k-1}\right) R^{m}[x]$ into itself.

For the reparameterization, consider the polynomial-valued function $F(h)$ given by

$$
F(h)(x) \stackrel{\text { def }}{=} J_{k}\left\{\left[I_{m}+\Psi(h)(x)\right] \cdot f(x+\Phi(h)(x))-f(x)\right\} .
$$

This map has the four properties. It is analytic. It maps the polynomial space $\left(J_{k}-J_{k-1}\right) R^{m}[x]$ into the same space. It fixes the origin $h=0$ of this space. At the origin $h=0$ its $h$-Fréchet derivative is the identity map on the space $\left(J_{k}-J_{k-1}\right) R^{m}[x]$. These properties are established below after their application. The application is next.

Let $z=z(x)$ also denote a homogeneous polynomial of degree $k$ in the space $\left(J_{k}-J_{k-1}\right) R^{m}[x]$. The single equation or polynomial identity $F(z)(x)=$ $h(x)$ stands for an finite number of simulataneous equations with analytic or polynomial dependence on the coefficients of $z(x)$ and $h(x)$. These simultaneous equations are obtained by equating coefficients of monomials $x^{\alpha}$ when $|\alpha|=k$. Given the four properties, the finite-dimensional, implicit function theorem applied to the equation $F(z)=h$ yields an analytic function $z(h)(\cdot)$ valued in polynomial space $\left(J_{k}-J_{k-1}\right) R^{m}[x]$ with $z(0)(x)=0$ identically, so the origin of $\left(J_{k}-J_{k-1}\right) R^{m}[x]$ is fixed, and such that $F(z(h))=h$ whenever $\|h\|$ is sufficiently small. The latter says

$$
J_{k}\left\{\left[I_{m}+\Psi(z(h))(x)\right] \cdot f(x+\Phi(z(h))(x))\right\}=J_{k} f(x)+h(x) .
$$

The analytic polynomial-valued function $z(h)(\cdot)$ of the coefficients of $h$ provides the reparameterization needed to meet the conclusions of the lemma in the case $j=0$.

The following demonstrations of the four properties complete this step and end the proof of the lemma.

The map is analytic for the following reasons. The polynomial-valued map $F(h)(\cdot)$ is obtained from the composition of analytic polynomial-valued functions of the coefficients of the polynomial $h$. The appearance of $C^{k}$ map $f$ in the definition of $F(h)(x)$ can be replaced by its $k$-jet $p(x)=J_{k} f(x)$. In consequence, the cofficients of the polynomial-valued map $F(h)(x)$ depend analytically on the coefficients of the homogeneous polynomial $h$.

The origin of $\left(J_{k}-J_{k-1}\right) R^{m}[x]$ is a fixed point of the map $F$. That is, $F(0)(x)=0$, since by Steps B and C, $(A(1)(x), Y(1)(x))=\left(I_{m}, x\right)$ when $h=0$ is the identically zero polynomial.

The space $\left(J_{k}-J_{k-1}\right) R^{m}[x]$ is mapped into itself due to the following. For each $h$ in $\left(J_{k}-J_{k-1}\right) R^{m}[x]$, the polynomial $F(h)(x)$ by its definition has degree $k$ in the indeterminates $x=\left(x_{1}, \ldots, x_{n}\right)$. By the calculations in Step 
D until the assumption $j>0$ is utilised,

$$
\begin{aligned}
F(h)(x) & =\int_{0}^{1} \frac{d}{d t} J_{k}[A(t)(x) \cdot f(Y(t)(x))] d t \\
& =\int_{0}^{1} J_{k}[A(t)(x) \cdot[h] \circ Y(t)(x)] d t .
\end{aligned}
$$

Now the integrand is a $t$-dependent polynomial curve. From $Y(t)(x)=O(|x|)$, the integrand $h \circ Y(t)(x)=h(Y(t)(x))=O\left(|Y(t)(x)|^{k}\right)=O\left(|x|^{k}\right)$ lies in the vector space $\left(J_{k}-J_{k-1}\right) R^{m}[x]$. Thus for each $h$, the polynomial $F(h)(x)$ belongs to the subspace $\left(J_{k}-J_{k-1}\right) R^{m}[x]$ as required.

Analyticity allows the required Fréchet derivative to be obtained from a directional derivative. At $h$ in $\left(J_{k}-J_{k-1}\right) R^{m}[x]$, the Fréchet derivative $D_{h} F(0)(\cdot)$ is provided by the following calculation.

$$
\begin{aligned}
\left\{D_{h} F(0)\right. & \cdot h\}(x)=\left.\frac{d}{d t}\right|_{t=0}[F(t \cdot h)(x)] \\
& =\left.\frac{d}{d t}\right|_{t=0} J_{k}\left\{\left[I_{m}+\Psi(t h)(x)\right] \cdot f(x+\Phi(t h)(x))-f(x)\right\} \\
& \left.=\left.\frac{d}{d t}\right|_{t=0} J_{k}\{A(t)(x)] \cdot f(Y(t)(x))\right\}-\left.\frac{d}{d t}\right|_{t=0} J_{k}\{f(x)\} \\
& =J_{k}\left\{A^{\prime}(0)(x) \cdot p(Y(0)(x))+A(0)(x) \cdot\left(D_{x} p\right)(Y(0)(x)) \cdot Y^{\prime}(0)(x)\right\} \\
& =J_{k}\left\{\left[J_{k} A(0)(x) a(h)(Y(0)(x))\right] p(Y(0)(x))\right. \\
& \left.\quad+A(0)(x)\left(D_{x} p\right)(Y(0)(x))\left[J_{k} w(h)(Y(0)(x))\right]\right\} \\
& =J_{k}\left\{\left[J_{k} I_{m} \cdot a(h)(x)\right] \cdot p(x)+I_{m} \cdot\left(D_{x} p\right)(x) \cdot\left[J_{k} w(h)(x)\right]\right\} \\
& =J_{k}\left\{\left[J_{k} a(h)(x)\right] \cdot p(x)+\left(D_{x} p\right)(x) \cdot\left[J_{k} w(h)(x)\right]\right\} \\
& =h(x) \quad
\end{aligned}
$$

by the properties of $(a(h)(x), w(h)(x))$ in Step A. The proof is complete.

Proof of Lemma 4.2. Take the linear, polynomial map $a(h)(x)$ to be identically zero in Step A. This entails in step C that $A(t)(x)=I_{m}=1$ identically, and hence $\Psi(h)(x)=A(1)(x)-I_{m}=0$ as required in Lemma 4.1.

\section{APPENDIX: LIE GROUP ASPECTS OF THE POLYNOMIAL CALCULATIONS}

Let $G$ be the collection of elements $(A(x), Y(x))$ in the the product space $J_{k} R^{m \times m}[x] \times\left(J_{k}-J_{0}\right) R^{n}[x]$ such that $A(0)$ and $D_{x} Y(0)$ are nonsingular $m \times m$ and $n \times n$ matrices respectively. Note $Y(0)=0$ as $\left(J_{k}-J_{0}\right) R^{n}[x]$ consists of all polynomial maps $Y: R^{n} \rightarrow R^{m}$ such that $Y(x)=O(|x|)$. The product of two elements $(A(x), Y(x))$ and $(B(x), Z(x))$ in $G$ is say

$$
(A(x), Y(x)) \cdot(B(x), Z(x)) \stackrel{\text { def }}{=}\left(J_{k}[A(x) \cdot B(Y(x))], J_{k}[Z(Y(x))]\right) \text {. }
$$

This product takes values in $G$. The product is associative. Equipped with this product, $G$ forms a Lie group. Its identity element is $1=(A(x), Y(x))=$ $\left(I_{m}, x\right)$. The multiplicative inverse of $(A(x), Y(x))$ exists and is given by 
$\left(J_{k}\left[A(x)^{-1}\right], J_{k}\left[Y^{-1}(x)\right]\right)$. The matrix inverse $A(x)^{-1}$ of the $A(x)$ can be calculated by Cramer's rule. Its components are polynomials in $x$ divided by the polynomial $\operatorname{det}[A(x)]$. The latter of course is nonzero at $x=0$ since $A(0)$ is nonsingular. The components of the matrix inverse are thus analytic functions of $x$ defined on a common neighbourhood of the origin $x=0$ in $R^{n}$. So the $k$-jet operator can be applied. Similarly, the inverse $Y^{-1}(x)$ of the polynomial map $Y(x)$ is an analytic map on a neighbourhood of the origin $x=0$ in $R^{n}$ with $Y^{-1}(0)=0$. So its $k$-jet is also defined. The components of the polynomials $\left(J_{k}\left[A(x)^{-1}\right], J_{k}\left[Y^{-1}(x)\right]\right)$ are polynomial expressions in those of $(A(x), Y(x))$. Thus $G$ is a Lie group.

Given a polynomial (or $C^{k}$ ) map $f: R^{n} \rightarrow R^{m}$, the action of an element $(A(x), Y(x))$ in $G$ on the $k$-jet $J_{k} f(x)$ is given by

$$
(A(x), Y(x)) \cdot f(x)=J_{k}[A(x) \cdot f(Y(x))] .
$$

The orbit $G \cdot J_{k} \hat{f}$ is a submanifold of the polynomial space $J_{k} R^{m}[x]$. This orbit passes through the $k$-jet $J_{k} f(x)$. The tangent space at $J_{k} f(x)$ of this orbit consists of all elements of the form

$$
L(a, w)(x)=J_{k}[a(x) \cdot f(x)]+D_{x}\left[J_{k} f(x)\right] \cdot w(x)
$$

where $(a(x), w(x))$ belong to the tangent space

$$
T_{1} G=J_{k} R^{m \times m}[x] \times\left(J_{k}-J_{0}\right) R^{n}[x]
$$

of $G$.

Let $G_{0}=G$. For $0<j<k$, the set

$$
G_{j}=\left\{I_{m}+\left(J_{k}-J_{j}\right) R^{m \times m}[x]\right\} \times\left\{x+\left(J_{k}-J_{j}\right) R^{n}[x]\right\}
$$

is a subset of $G$. Then for $0 \leq j<k$, the sets $G_{j}$ are proper or improper Lie subgroups of the Lie group $G_{0}=G$. Each algebraic conditions $A C_{k . j}(f)$ say that the tangent space at the origin $J_{k} f$ of the orbit $G_{j} \cdot J_{k} f$ includes the linear space $\left(J_{k}-J_{k-1}\right) R^{m}[x]$ of homogeneous polynomial maps $h(x)=$ $\sum_{|\alpha|=k} h_{\alpha} \cdot x^{\alpha}$ of $R^{n}$ into $R^{m}$. Lemma 4.2 implies there is a submanifold $M_{j}$ of each $G_{j}$ passing through the common identity element of $G$ and $G_{j}$ such that $M_{j} \cdot J_{k} f(x)$ is a neigbourhood of $J_{k} f(x)$ in the linear manifold $J_{k} f(x)+\left(J_{k}-J_{k-1}\right) R^{m}[x]$ of $k$ th-order polynomial perturbations of the $k$ jet $J_{k} f(x)$. The polynomial calculations in the proof of Lemma 4.2 can be expressed in terms of exponential maps on the Lie algebras of the groups $G_{j}$.

For $(a(x), w(x))$ in the tangent space of

$$
T_{1} G=J_{k} R^{m \times m}[x] \times\left(J_{k}-J_{0}\right) R^{n}[x]
$$

of $G_{j}$, a left invariant vector field on $G_{j}$ is given by

$$
\vec{V}_{(a, w)}(A, Y)=\left(J_{k} A(x) \cdot a \circ Y(x), J_{k} w \circ Y(x)\right) .
$$


The exponential map on the lie algebra of $G_{j}$ is denoted here by EXP. The exponential map EXP at $\vec{V}_{(a, w)}$ in the tangent space

$$
T_{1} G_{j}=\left\{\left(J_{k}-J_{j}\right) R^{m \times m}[x]\right\} \times\left\{\left(J_{k}-J_{j}\right) R^{n}[x]\right\}
$$

is determined by the time 1 map of the initial value problem

$$
\begin{array}{cc}
\frac{d B(t)}{d t}(x)=J_{k}[B(t)(x) \cdot a(Z(t)(x))], & B(0)(x)=I_{m}, \\
\frac{d Z(t)}{d t}(x)=J_{k}[w(Z(t)(x))], & Z(0)(x)=x .
\end{array}
$$

The two equations in this initial value problem are equations (ODE2) and (ODE1) met earlier in Steps $\mathrm{C}$ and $\mathrm{B}$ respectively. Here for all real $t$, $\operatorname{EXP}\left(t \cdot V_{(a, w)}\right)(x)=(B(t)(x), Z(t)(x))$ is a polynomial of degree $k$ in $x$ which has an analytic dependence on $t$ and the coefficients of the polynomials $(a(x), w(x))$. Further, for $s$ and $t$ real, the exponential property

$$
\operatorname{EXP}\left(t \cdot V_{(a, w)}\right)(x) \cdot \operatorname{EXP}\left(s \cdot V_{(a, w)}\right)(x)=\operatorname{EXP}\left((t+s) \cdot V_{(a, w)}\right)(x)
$$

holds.

Verification of the exponential property follows from the theory of ordinary differential equations. The ordinary differential equations in the above initial value problem is associated with a left-invariant vector-field on each of the Lie groups $G_{j}$.

In the case of right determinacy, the foregoing considerations simplify. Take $\widetilde{G}$ to be the Lie group consisting of polynomials maps $Y(x)$ in the space $\left(J_{k}-J_{0}\right) R^{n}[x]$ such that the Jacobian matrix $D_{x} Y(0)$ is nonsingular. The group operation is composition followed by truncation of terms of order $k+1$ or greater. The exponential map EXP at $w$ in the tangent space $T_{1} \widetilde{G}=$ $\left(J_{k}-J_{0}\right) R^{n}[x]$ is given by $\operatorname{EXP}\left(t \cdot \vec{V}_{w(\cdot)}\right)=Z(t)(x)$ where

$$
\frac{d Z(t)}{d t}(x)=J_{k} w(Z(t)(x)), \quad Z(0)(x)=x .
$$

Here $\vec{V}_{w(\cdot)}(y)=J_{k}[w(y(x))]$ defines a left-invariant vector field on $\tilde{G}$.

\section{Polynomial RePresentation of MAPS}

Univariate Taylor's formula. Let $m, n$ and $k$ be positive integers as before. Let $g(q)$ be a $C^{k}$ real- or $R^{m}$-valued function of a real variable $q$ in the closed interval $[0,1]$. Then the univariate form of Taylor's formula says

$$
g(1)=\sum_{i=0}^{k} \frac{1}{i !} g^{(i)}(1)+R(g)
$$

where an integral representation of the remainder

$$
R(g)=\int_{0}^{1} \frac{(1-q)^{k-1}}{(k-1) !}\left[g^{(k)}(q)-g^{(k)}(0)\right] d q
$$


The $i$ th derivative of the function $g(q)$ is denoted by $g^{(i)}(q)$. The zeroth derivative $g^{(0)}(q) \stackrel{\text { def }}{=} g(q)$ is the original function.

Multivariate Taylor's formula. Let $h: R^{n} \rightarrow R^{m}$ be a $C^{k}$ or smoother map defined on a convex neighbourhood of the origin or a domain starred with respect to the origin. For each $x$ in $a$ the domain of $h$, the $C^{k}$ function $g(q)=h(q x)$ of the single real variable $q$ in the interval $[0,1]$ satisfies $g(1)=h(x)$. The univariate Taylor formula, the chain-rule, and the multinomial formula together imply the multivariate Taylor formula

$$
\begin{aligned}
h(x) & =g(1)=\sum_{i=0}^{k} \frac{1}{i !} g^{(i)}(1)+R(g) \\
& =\left.\sum_{i=0}^{k} \frac{1}{i !}\left(\frac{d}{d q}\right)^{i} h(q x)\right|_{q=1}+R(g) \\
& =\sum_{i=0}^{k} \frac{1}{i !}\left[\sum_{|\alpha|=i}\left(\begin{array}{l}
i \\
\alpha
\end{array}\right) \partial^{\alpha} h(0) \cdot x^{\alpha}\right]+R(g) \\
& =\left[J_{k} h\right](x)+R(g) .
\end{aligned}
$$

The integral representation of the remainder term becomes

$$
R(g)=\int_{0}^{1} \frac{(1-q)^{k-1}}{(k-1) !} \sum_{|\alpha|=k}\left(\begin{array}{l}
k \\
\alpha
\end{array}\right)\left[\partial^{\alpha} h(q x)-\partial^{\alpha} h(0)\right] \cdot x^{\alpha} d q
$$

The representation and its properties. For every point $t=\left(t_{1}, \ldots, t_{n}\right)$ in the (convex) domain of $h$, let

$$
[Q(t) h](x) \stackrel{\text { def }}{=}\left[J_{k} h\right](x)+\sum_{|\alpha|=k} \frac{1}{k !}\left(\begin{array}{l}
k \\
\alpha
\end{array}\right) \int_{0}^{1} \frac{(1-q)^{k-1}}{(k-1) !}\left[\partial^{\alpha} h(q t)-\partial^{\alpha} h(0)\right] d q \cdot x^{\alpha} \text {. }
$$

The operator $Q(t)$ has several properties.

First, the map reproducing property, $[Q(t) h](t)=h(t)$ follows immediately from Taylor's formula.

Second, $[Q(t) h](x)$ is a polynomial of degree $k$ in $x$ with coefficients depending at least continuously on the auxiliary parameter $t$ and the function $h$. In the event that $h$ lies in a second polynomial space $J_{K} R^{m}[x]$ where $K>k$, the coefficients of $[Q(t) h](x)$ are themselves polynomial expressions in $t$ and the coefficients of $h$. The dependence on $h$ is linear. The $t$-dependence is $C^{u-k}$ when $h$ is $C^{u}$.

Third, $Q(0) h=J_{k} h$. So $[Q(t) h](x)$ belongs to the linear manifold $\left[J_{k} h\right]+$ $\left(J_{k}-J_{k-1}\right) R^{m}[x]$. The polynomial $[Q(t) h](x)$ is therefore an $O\left(|x|^{k}\right), t$ dependent, perturbation or disturbance of the polynomial $k$-jet $[Q(0) h](x)=$ $\left[J_{k} h\right](x)$.

Fourth, adopt the convention that the operators $Q(t)$ and $J_{k}$ act only on the $x$-dependence of any map or polynomial. Thus $Q(t)$ applied to the $t$ dependent polynomial $[Q(t) h](x)$ ignores the $t$-dependence of the latter, and 
yields $[Q(t)[Q(t) h]](x)=[Q(t) h](x)$. For $t$ fixed, the operator $Q(t)$ is a projection: $[Q(t)]^{2}=Q(t)$. It projects each $C^{k}$ or smoother map $h(x): R^{n} \rightarrow$ $R^{m}$ into a $t$-determined element $[Q(t) h](x)$ in the polynomial space $J_{k} R^{m}[x]$.

For each function $h(z, x)$ of two variables which for fixed $z$ is at least $k$-times continuously differentiable with respect to the variable $x$, we obtain a $z$-dependent family $H(z)(\cdot)$ of functions $H(z)(x)=h(z, x)$ on which the operator $Q(t)$ acts. $[Q(t) H(z)(\cdot)](x)=[Q(t) h(z, \cdot)](x)$ is a family of $(z, t)-$ dependent polynomials in $x$ with the property that the substitution $x=t$ yields $h(z, t)$.

Finally, consider a map $h(t, x)$ with $C^{k}$ dependence on $x$ when $t$ is fixed. This map yields a family of $t$-determined $C^{k}$ functions $H(t)(\cdot)$ defined at $x$ by $H(t)(x)=h(t, x)$. For each $t, Q(t)$ acts on the $x$-dependence only of the function $H(t)(x)$. The resulting family of $t$-dependent polynomials $[Q(t) H(t)(\cdot)](x)=[Q(t) h(t, \cdot)](x)$ in $J_{k} R^{m}[x]$ now depends on $t$ in $R^{n}$ due to (i) the original dependence of $h(t, x)$ on $t$ and (ii) the definition of the operators $Q(t)$. Further for each $t$, the substitution $x=t$ into the $t$-determined polynomial $[Q(t) H(t)(\cdot)](x)=[Q(t) h(t, \cdot)](x)$ produces the $t$ determined function $H(t)(\cdot)=h(t, \cdot)$ of $x$ evaluated at the point $x=t$ in $R^{n}$. That is, the substitution $x=t$ yields $\left.[Q(t) h(t)(\cdot)](x)\right|_{x=t}=\left.H(t)(x)\right|_{x=t}=$ $h(t, t)=$ the diagonal value of the map $h(t, x)$ at $(x, t)=(t, t)$.

Remark A. In the proof of Theorem 3.1, a map $h(t, x)=A(t, x) \cdot f(y(t, x))$ appears. The function $f$ here is at least $C^{u}$ where $u \geq k . A(t, x)$ denotes an $m \times m$ square matrix. The map $y(t, x)$ takes values in $R^{n}$. Both $A(t, x)$ and $y(t, x)$ are given by polynomials in $x$ with $t$-dependent coefficients. The diagonal values of the map $h(t, x)$ are $h(t, t)=A(t, t) \cdot f(y(t, t))$. Thus

$$
\left.[Q(t) h(t)(\cdot)](x)\right|_{x=t}=\left.[Q(t) A(t, \cdot) \cdot f(y(t, \cdot))](x)\right|_{x=t}=A(t, t) \cdot f(y(t, t)) .
$$

The employment of this diagonal-value reproducing property is vital. The matrix-valued function and the change of variables required in the conclusions of Theorem 3.1 are given by the diagonal values $A(t, t)$ and $y(t, t)$ of $t$ dependent polynomials $A(t, x)$ and $y(t, x)$ respectively.

Remark B. A parameter-dependent representation of sufficiently differentiable functions is implicit in the proofs of the Morse lemma in Martinet [1982] and Hormander [1971] (or Nirenberg [1973-1974]). In these proofs, $C^{2}$ functions $h$ with a Morse singularity at the origin of $R^{n}$ are identified with symmetric $n \times n$ matrix-valued maps. If $k=2$, the matrix-valued functions provide the coefficients of a $t$-dependent, quadratic polynomial $Q(t) h[x]$. These proofs of the Morse lemma illustrate the diagonal-value reproducing property.

\section{Proof of Theorem 3.1}

Let $z(x)$ and $h(x)$ denote elements of the space $\left(J_{k}-J_{k-1}\right) R^{m}[x]$ of homogeneous polynomials of order and degree $k$ in $x$ with values in $R^{m}$. Let 
$z$ replace $h$ in the statement of Lemma 4.1 to obtain polynomial-valued maps $\Phi(z)(x)$ and $\Psi(z)(x)$ with analytic dependence on the coefficients of the polynomial $z$. These maps satisfy the $k$ th-order approximation property

$$
J_{k} f(x)+z(x)=J_{k}\left\{\left[I_{m}+\Psi(z)(x)\right] \cdot f(x+\Phi(z)(x))\right\}
$$

for $\|z\|<\varepsilon_{j}$ where $0<\varepsilon_{j} \leq \infty$. This polynomial identity gives to order $k$, the conclusions of Theorem 3.1.

Let $\operatorname{Id}(x)=x$ denote the identity map on $R^{n}$. The application of the $Q(t)$ operator to the $C^{u}$ map

$$
\left(I_{m}+\Psi(z)(x)\right) \cdot f(\operatorname{Id}(x)+\Psi(z)(x))-f(x)
$$

yields a polynomial-valued map

$$
F(t, z)(x) \stackrel{\text { def }}{=}\left[Q(t)\left\{\left(I_{m}+\Psi(z)(\cdot)\right) f \circ(\mathbf{I d}+\Psi(z)(\cdot))-f(\cdot)\right\}\right](x) .
$$

The coefficients of the polynomial $F(t, z)(x)$ have a $C^{u-k}$ dependence on the coefficients of the polynomial $z(x)$ and the $R^{n}$-valued, variable $t$. Here $Q(0)=J_{k}$ and the $k$ th-order polynomial approximation (1) imply $F(0, z)(x)$ $=z(x)$. So $F(0, z)$ belongs to the polynomial space $\left(J_{k}-J_{k-1}\right) R^{m}[x]$. Now $F(t, z)=F(0, z)+O\left(|x|^{k}\right)$ for each fixed $t$. So $F(t, z)$ belongs to this polynomial space $\left(J_{k}-J_{k-1}\right) R^{m}[x]$ as well. Now $F(0,0)=0$ since $F(0, z)=z$. The latter also implies partial $z$-Fréchet of $F(0, z)$ is the identity map on the polynomial $\left(J_{k}-J_{k-1}\right) R^{m}[x]$. The hypotheses of the finite-dimensional, inverse function theorem are satisfied.

For a special case of the conclusion let $h(x)=O\left(|x|^{k}\right)$ first denote a polynomial in the space $\left(J_{k}-J_{k-1}\right) R^{m}[x]$. Its norm is say

$$
\|h\| \stackrel{\text { def }}{=} \max _{|\alpha|=k}\left\{\left|\frac{1}{\left(\alpha_{1}\right) ! \cdots\left(\alpha_{n}\right) !} \cdot \frac{\partial^{\alpha} h}{\partial x^{\alpha}}(0)\right|\right\} .
$$

Regard the equation $F(t, z)(x)=h(x)$ as a polynomial identity in $x$ with coefficients possessing a $C^{u-k}$ dependence on the coefficients of $z$ and $h$, and on the components of the variable $t$. By equating coefficients of the monomials $x^{\alpha}$ (where $|\alpha|=k$ ), this identity may further be viewed as a finite-dimensional system of $C^{u-k}$ equations involving $t$ and the coefficients of both $z(x)$ and $h(x)$.

By the $k$ th-order approximation property (1), the polynomial identity $F(t, z)(x)=h(x)$ holds at $h=z=0$ in $\left(J_{k}-J_{k-1}\right) R^{m}[x]$ when $t=$ 0 in $R^{n}$. Therefore the polynomial identity implicitly determines a $C^{u-k}$ polynomial-valued map $z(x)=z(h, t)(x)$ with $z(0, h)(x)=h(x)$ such that $F(z(h, t))(x)=h(x)$ whenever $\|t\|<a$ and $\|h\|<c$ for some positive numbers $a$ and $c$.

It is clear that $a$ can be diminished without changing $c$, and vice versa.

The polynomial identity $h(x)=F(z(h, t))(x)$ written in full stands for the equality

$$
h(x)=\left[Q(t)\left\{\left(I_{m}+\Psi(z(t, h))(\cdot)\right) f \circ(\mathbf{I d}+\Psi(z(t, h))(\cdot))-f(\cdot)\right\}\right](x) .
$$


The substitution $x=t$ in this polynomial identity yields

$$
h(t)=\left[I_{m}+\Psi(z(t, h))(t)\right] \cdot f([t+\Psi(z(t, h))(t)])-f(t)
$$

due to the map and diagonal-value reproducing properties of the $Q(t)$ operator discussed earlier. Let

$$
A(t, h) \stackrel{\text { def }}{=} I_{m}+\Psi(z(t, h))(t)
$$

and

$$
y(t, h) \stackrel{\text { def }}{=} t+\Phi(z(t, h))(t) .
$$

Then $A(t, h)$ and $y(t, h)$ have the form required in conclusion in the special case that $h$ is a polynomial since both $\Psi(z(t, h))(x)$ and $\Psi(z(t, h))(x)$ are polynomials of order $O\left(|x|^{j+1}\right)$. Their coefficients possess $C^{u-k}$ dependence on the components and coefficients of the arguments $(t, h)$. Finally, the foregoing definitions give

$$
A(t, h) \cdot f(y(t, h))=f(t)+h(t)
$$

as required, albeit with the variable $t$ in place of the variable $x$ and a polynomial in place of a $C^{u}$ map.

For the general case, assume $h(x)$ belongs to the complete, normed, function space $\left(1-J_{k-1}\right) C^{u}\left(B_{a}\left(R^{n}\right), R^{m}\right)$. Recall the latter space consists of $C^{u}, R^{n}$ valued, $O\left(|x|^{k}\right)$ (that is $(k-1)$-flat) maps defined on the ball $\|x\| \leq a$. Suppose the $C^{k}$ norm of $h$ satisfies

$$
c>\|h\|_{C^{k}} \stackrel{\text { def }}{=} \sup \left\{\frac{1}{\left(\alpha_{1}\right) ! \cdots\left(\alpha_{n}\right) !} \cdot \frac{\partial^{\alpha} h}{\partial x^{\alpha}}(x):\|x\| \leq a \text { and } 0 \leq|\alpha| \leq k\right\} .
$$

Then for each $t$ satisfying $\|t\| \leq a$, the norm of the polynomial $[Q(t)] h(x)$ in the space $\left(J_{k}-J_{k-1}\right) R^{m}[x]$ is also less than $c$. This follows from Taylor's formula and the definition of the operator $Q(t)$. Now $z(t,[Q(t)] h)$ is defined and

$$
A(t,[Q(t)] h) \cdot f(y(t, Q(t) h))=f(t)+[Q(t) h](t)=f(t)+h(t)
$$

for $\|t\| \leq a$. This yields the required changes of variables and matrix-valued functions in the conclusion of Theorem 3.1 after an obvious change of notation. The required properties (I), (III) and (IV) follow immediately.

The forms of

$$
y(t,[Q(t) h])=t+\left.\Phi(z(t,[Q(t) h]))(x)\right|_{x=t}
$$

and

$$
A(t,[Q(t) h])=I_{m}+\left.\Psi(z(t,[Q(t) h]))(x)\right|_{x=t}
$$

imply their respective $t$-Fréchet derivatives exist at $t=0$ even if $u=k$. Further at $t=0$ the partial $t$-Fréchet derivative

$$
\left.D_{t} y(t,[Q(t) h])\right|_{t=0}=I_{n}+\left.D_{x}(\Phi(z(0,[Q(t) h]))(x))\right|_{x=0}=D_{x} Y(1)(x) \text {. }
$$


This partial $t$-Fréchet derivative is nonsingular by the substep ending Step B in the proof of Lemma 4.1. Hence $D_{t} y(t,[Q(t) h])$ is nonsingular at $t=0$ as required. The foregoing yields property (V). The matrix $A(t,[Q(t)] h)=$ $I_{m}+\Psi(z(t,[Q(t)] h))(t)$ is also nonsingular at $t=0$ by the substep ending Step $\mathrm{C}$ in the proof of Lemma 4.1. (Alternatively, note $A(t,[Q(t) h]$ ) is a perturbation of the identity matrix $I_{m}$ with continuous dependence on $(t, h)$. Hence it is nonsingular if $a$ and $c$ are sufficiently small.) The preceding gives property (II). So Theorem 3.1 is proven.

\section{ACKNOWLEDGEMENTS.}

In writing this article and the thesis on which it was based I enjoyed the assistance of several people. My graduate studies at McGill University were supervised by W. F. Langford. In my thesis work a right determinacy result included in Theorem 3.1 (the case $j=0$ ) was established but for the existence of the submanifold provided by Lemma 4.2. Thinking the existence might follow from some Lie group theory, I approached Carl Herz for advice. The advice completed the missing step. After the advice was given, I met the text Martinet [1982]. It contains all the arguments needed for a proof of a right determinacy result except for an explicit polynomial representation of sufficiently differentiable maps. The forerunner of Theorem 3.2 on the loss of fewer derivatives was discussed between Vũ-Trong-Tuân, Carl Herz and myself. The generalization here of the treatment of right determinacy in my doctoral dissertation Selby [1983] to a treatment of both right and contact determinacy follows an observation on its feasibility by the referee. James Healey of the English Department at Eastern Montana College and the referee further helped in the correction of typographical and other errors in draft versions of this article.

\section{REFERENCES}

For more comprehensive bibliographies prior to 1980 , see the survey paper Wall [1981] or the elementary text Poston and Stewart [1978].

1. R. Abraham and J. Robbin, Transversal mappings and flows, Benjamin, New York, 1967.

2. D. D. Ang and Vũ-Trong-Tuân, A representation theorem for differentiable functions, Proc. Amer. Math. Soc. 75 (1979), 343-350.

3. V. I. Arnold, Singularity theory, Selected Papers from 1968 to 1979, London Math. Soc. Lecture Notes 53, Cambridge Univ. Press, 1981.

4. V. I. Balatin, Topological equivalence of germs of functions, Mat. Zametki 35 (1984), 579-588 [Math. Notes 35 (1984), 306-310].

5. R. G. Bartle, Singular points of functional equations, Trans. Amer. Math. Soc. 75 (1953), 366-384.

6. G. R. Belickii, Equivalence and normal forms of germs of smooth maps, Uspehi Mat. Nauk 33 (1978), 1(199), 95-155, 263 [Russian Math. Surveys 33 (1978), no. 1, 107-177].

7. M. Berger, Nonlinearity and functional analysis: lectures on nonlinear problems in mathematical analysis, Academic Press, New York, 1977.

8. J. Bochnak and W. Kucharz, Sur les germes d'applications différentiables à singularités isolées, Trans. Amer. Math. Soc. 252 (1979), 115-131. 
9. J. W. Bruce, On the degree of determinacy of smooth functions, Bull. London Math. Soc. 13 (1981), 55-58.

10. J. W. Bruce, T. Gaffney and A. A. du Plessis, On left equivalence of map germs, Bull. London Math. Soc. 16 (1984), 303-306.

11. Th. Bröcker and L. Lander, Differentiable germs and catastrophes, Cambridge Univ. Press, Cambridge, 1975.

12. M. A. Bruce, A note on $C^{1}$ equivalence, J. Math. Anal. Appl. 121 (1987), 91-95.

13. M. Buchner, J. Marsden and S. Schecter, Application of the blowing-up construction and algebraic topology, J. Differential Equations 48 (1983), 404-433.

14. H. Cartan, Elementary theory of analytic functions of one or several variables, Addison-Wesley, London, 1963.

15. P. Cragnolini and A. Milani, Topologies of deformations, Boll. Un. Mat. Ital. 3 (1984), 883893.

16. J. Damon, The unfolding and determinacy theorems for subgroups of $\mathscr{A}$ and $\mathscr{K}$, Singularities (Arcata, Calif., 1981), Proc. Sympos. Pure Math., vol. 40, Part 1, Amer. Math. Soc. 1983, Providence, R. I., pp. 233-254.

17. $\ldots$ The unfolding and determinacy theorems for subgroups of $\mathscr{A}$ and $\mathscr{K}$, Mem. Amer. Math. Soc. No. 306 (1984), 90 pp.

18. _ Topological properties of real simple germs, curves, and the nice dimensions $n>p$, Math. Proc. Cambridge Philos. Soc. 89 (1981), 451-472.

19. A. B. Deakin, New proofs of some theorems on infinitely differentiable functions, Bull. Austral. Math. Soc. 17 (1977), 161-175.

20. _ The formal power series approach to elementary catastrophe theory, Contemp. Math., vol. 9, Amer. Math. Soc., Providence, R. I., 1981, pp. 313-317.

21. _ The formal power series approach to elementary catastrophe theory, Bull. Math. Biol. 40 (1978), 429-450.

22. N. Desolneux-Moulis, Sufficiency of jets of maps from a Hilbert space to the real line, Global Analysis and its Applications, vol. III, Trieste, 1972, Internat. Atomic Energy Agency, Vienna, 1974.

23. Takuo Fukuda, Local topological properties of differentiable mappings, I, Invent. Math. 65 (1981-82), 227-250.

24. T. Gaffney, More on the determinacy of smooth map germs, Invent. Math. 66 (1982), 137-163.

25. J. J. Gervais, On right-left $C^{\infty}$ sufficiency of jets, C. R. Math. Rep. Acad. Sci. Canada 4 (1982), 341-346.

26. C. Gibson, G. Wirthmüller, A. A. du Plessis, and E. Looijenga, Topological stability of smooth mappings, Lecture Notes in Math., vol. 552, Springer, 1977.

27. G. Glaeser, Fonctions composées différentiable, Ann. of Math. 77 (1963), 193-206.

28. M. Golubitsky and V. Guillemin, Stable mappings and their singularities, Springer, New York, 1973.

29. M. Golubitsky and D. Schaeffer, A theory of imperfect bifurcation via singularity theory, Comm. Pure Appl. Math. 43 (1979), 21-98.

30. E. P. Gomozov, Equivalence of germs of smooth mappings with respect to transformations of coordinates on the pre-image, Studies in the Theory of Functions of Several Variables, Yaroslav Gos. Univ., Yaroslavl, 1980. (Russian)

31. _ - Versatility theorems for some groups of coordinate transformations, Manifolds of Differentiable Mappings (P. W. Michor, editor), Shiva Math. Series, Birkhäuser-Verlag, 1980.

32. M. L. Graves, Remarks on singular points of functional equations, Trans. Amer. Math. Soc. 79 (1955), 150-157.

33. J. K. Hale, Ordinary differential equations, Wiley, New York, 1969.

34. P. Hartman, Ordinary differential equations, Wiley, New York, 1964. 
35. E. Hille and R. S. Philips, Functional analysis and semigroups, Amer. Math. Soc. Colloq. Publ., vol. 21, Amer. Math. Soc., Providence, R. I., 1957.

36. L. Hormander, Fourier integral operators, II. Acta Math. 127 (1971), 71-183.

37. F. Ichikawa, On finite determinacy of formal vector fields, Invent. Math. 70 (1982-83), 45-52.

38. F. Ichikawa, Generalized splitting for map-germs, Proc. Japan Acad. Ser. A Math. Sci. 59 (1983), 422-424.

39. M. C. Irwin, On the smoothness of the composition map, Quart. J. Math. 2 (1973), 113-133.

40. S. Izumiya, Stability of unfoldings, Hokkaido Math. J. 9 (1980), 36-45.

41. N. Kuiper, $C^{1}$ Equivalence of functions near a critical point, Proc. Sympos. Infinite Dimensional Topology (Baton Rouge, 1967), Ann. of Math. Studies, no. 69, Princeton Univ. Press, Princeton, N. J., 1972.

42. T. C. Kuo, On $C^{0}$-sufficiency of jets, Topology 8 (1969a), 167-171.

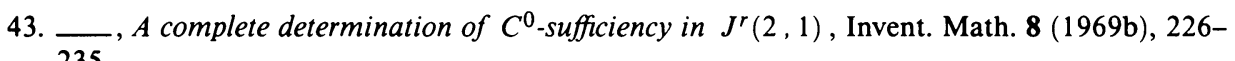
235.

44. __ Some viewpoints on algebraic geometry and singularity theory, Math. Chronicle 11 (1982), 67-80.

45. T. C. Kuo and Y. Lu, Sufficiency of jets via stratification theory, Invent. Math. 57 (1980), 219-226.

46. D. Lefebvre and M. T. Pourprix, Determinacy of $C^{\mu}$ map germs with respect to a $C^{q}$ contact equivalence, J. London Math. Soc. (2) 29 (1984), 367-379.

47. R. J. Magnus, The transformation of vector-functions, scaling and bifurcations, Trans. Amer. Math. Soc. 286 (1984), 689-713.

48. B. Malgrange, Ideals of differentiable functions, Oxford Univ. Press, Cambridge, 1966.

49. J. Martinet, Singularités des fonctions et applications différentiables, Lecture Notes, PUC, Rio de Janeiro, 1974.

50. __ Singularities of smooth functions and maps, London Math. Soc. Lecture Notes 58, Cambridge Univ. Press, 1982.

51. J. N. Mather, Stability of $C^{\infty}$ mappings I: The division theorem, Ann. of Math. 87 (1968), 89-104.

52. __ Right equivalence, Unpublished notes, Math. Institute Univ. of Warwick, Warwick, 1969a.

53. _ Stability of $C^{\infty}$ mappings. II: Infinitesimal stability implies stability, Ann. of Math. 89 (1969b), 254-291.

54. __ Stability of $C^{\infty}$ mappings. III: Finitely determined map germs, Publ. Math. Inst. Hautes Études Sci. Publ. Math. 35 (1968), 127-156.

55. __ Stability of $C^{\infty}$ mappings. IV: Classification of stable germs by $R$-algebras, Math. Inst. Hautes Études Sci. Publ. Math. 35 (1969), 223-248.

56. __ Stability of $C^{\infty}$ mappings. 'V: Transversality, Adv. in Mat. 4 (1970), 301-336.

57. __ Stability of $C^{\infty}$ mappings. VI: The nice dimensions, Proc. Liverpool Sympos. I, Lecture Notes in Math., vol. 192, Springer, 1970, pp. 207-253.

58. I. Nakai, Topological types of polynomial map germs, Singularities (Arcata, Calif., 1981), Proc. Sympos. Pure Math., vol. 40, Part 2, Amer. Math. Soc., Providence, R. I., 1983, pp. 227-231.

59. A. H. Nayfeh, Perturbation methods, Wiley, New York, 1973.

60. E. Nelson, Topics in dynamics I: Flows, Math. Notes, Princeton Univ. Press, Princeton, N. J., 1969.

61. L. Nirenberg, Topics in nonlinear functional analysis, Courant Inst. Lecture Notes, New York, 1973-74.

62. A. Orlandi, Classification relative des germes $f: R^{n} \rightarrow R$, Boll. Un. Mat. Ital. D 1 (1982), 193-214. 
63. R. Palais, The Morse lemma on Banach spaces, Bull. Amer. Math. Soc. 75 (1969), 968-971.

64. P. B. Percell and P. N. Brown, Finite determination of bifurcation problems, SIAM J. Math. Anal. 16 (1985), 28-46.

65. A. A. du Plessis, On the determinacy of smooth map germs, Invent. Math. 58 (1980), 107-106.

66. _- Genericity and smooth finite determinacy, Singularities (Arcata, Calif., 1981), Proc. Sympos. Pure Math., vol. 40, Part 1, Amer. Math. Soc., Providence, R. I., 1983, pp. 295-312.

67. A. A. du Plessis and L. Wilson, On right equivalence, Math. Z. 190 (1985), 165-205.

68. V. Poenaru, Singularités $C^{\infty}$ en présence de symétrie, Lecture Notes in Math., vol. 510, Springer, 1975.

69. T. Poston and I. Stewart, Catastrophe theory and its applications, Pitman Press, London, 1978.

70. J. D. Randall, Topological sufficiency of smooth map germs, Invent. Math. 67 (1982), 117-121.

71. J. Robbin, On the existence theorem for differential equations, Proc. Amer. Math. Soc. 19 (1968), 1005-1006.

72. C. Sabbah, Le type topologique éclaté d'une application analytique, Singularities (Arcata, Calif., 1981), Proc. Sympos. Pure Math., vol. 40, Part 2, Amer. Math. Soc., Providence, R. I., 1983, pp. 433-440.

73. R. J. Sacker, A new approach to the perturbation theory of invariant surfaces, Comm. Pure Appl. Math. 18 (1965), 717-732.

74. A. M. Selby, Determinacy and unfolding for non-smooth maps, Doctoral Thesis, Report 83-19, Department of Mathematics and Statistics, McGill University, Montreal, 1983.

75. M. Shiota, Equivalence of differentiable mappings and analytic mappings, Inst. Hautes Études. Sci. Publ. Math. 54 (1981), 237-322.

76. __ Equivalence of differentiable functions, rational functions and polynomials, Ann. Inst. Fourier (Grenoble) 32 (1982), 167-204.

77. J. Stanislaw, On algebraic criteria for $k$-determinacy of germs of smooth functions on manifolds with boundary, Demonstratio Math. 15 (1982), 1113-1123.

78. F. Takens, $A$ note on sufficiency of jets, Invent. Math. 13 (1971), 225-231.

79. R. Thom, Stabilité structurelle et morphogènése, Benjamin, New York, 1972.

80. J. C. Tougeron, Idéaux de fonctions différentiables. I, Ann. Inst. Fourier (Grenoble) 18 (1968), 177-240.

81. __, Idéaux de fonctions différentiables, Ergebnisse der Mathematik 71, Springer, 1972.

82. Vũ-Trong-Tuân, A representation theorem for differentiable functions. II, preprint, 1987.

83. C. T. C. Wall, Editor, Proceedings of Liverpool symposium I, Lecture Notes in Math., vol. 192, Springer, 1970.

84. __, Are maps finitely determined in general?, Bull. London. Math. Soc. 11 (1979), 151-154.

85. __, Finite determinacy of smooth map-germs, Bull. London Math. Soc. 13 (1981), 481-539.

86. __ Notes on the classification of singularities, Proc. London Math. Soc. 48 (1984), 461-513.

87. G. Wasserman, Stability of unfoldings, Lecture Notes in Math., vol. 393, Springer, 1974.

88. L. C. Wilson, Jets with regular zeroes, Pacific J. Math. 93 (1981), 471-478.

89. Y. Yomdin, Some results on finite determinacy not requiring the explicit use of smoothness, Singularities (Arcata, Calif., 1981), Sympos. Pure Math., vol. 40, Part 2, Amer. Math. Soc., Providence, R. I., 1983, pp. 667-674.

90. Guo Bin Zhang, A necessary and sufficient condition for a $C^{\infty}$ function to be finitely determined, Adv. in Math. (Beijing) 12 (1983), no. 1, 53-56. (Chinese)

29-16th Street, Roxboro, Quebec, Canada H8Y IN8 